\title{
The Practice Guidelines for Primary Care of Acute Abdomen 2015
}

\author{
Toshihiko Mayumi ${ }^{1} \cdot$ Masahiro Yoshida $^{2} \cdot$ Susumu Tazuma $^{3}$ - Akira Furukawa ${ }^{4}$. \\ Osamu Nishii ${ }^{5}$ Kunihiro Shigematsu ${ }^{6}$ - Takeo Azuhata ${ }^{7}$ Atsuo Itakura ${ }^{8}$. \\ Seiji Kamei ${ }^{9}$ Hiroshi Kondo ${ }^{10}$ - Shigenobu Maeda ${ }^{11} \cdot$ Hiroshi Mihara $^{12}$. \\ Masafumi Mizooka $^{3}$ - Toshihiko Nishidate ${ }^{13}$ - Hideaki Obara ${ }^{14} \cdot$ Norio Sato $^{15}$. \\ Yuichi Takayama $^{16}$. Tomoyuki Tsujikawa ${ }^{17}$. Tomoyuki Fujii ${ }^{18}$. Tetsuro Miyata ${ }^{19}$. \\ Izumi Maruyama $^{20} \cdot$ Hiroshi Honda ${ }^{21} \cdot$ Koichi Hirata $^{22}$
}

Published online: 18 December 2015

(C) Japan Radiological Society, Japanese Society of Hepato-Biliary-Pancreatic Surgery, and Japan Primary Care Association 2015

\begin{abstract}
Background Since acute abdomen requires accurate diagnosis and treatment within a particular time limit to prevent mortality, the Japanese Society for Abdominal Emergency Medicine in collaboration with four other medical societies launched the Practice Guidelines for Primary Care of Acute Abdomen that were the first English guidelines in the world for the management of acute abdomen. Here we provide the highlights of these guidelines [all clinical questions
\end{abstract}

The Practice Guidelines for Primary Care of Acute Abdomen 2015 were published in Japanese in 2015. Since these are first practical guidelines for acute abdomen and to inform more medical personnel in the world to improve medical practice and patient outcome, here we provide these guidelines in English. This guideline was developed in collaboration with the Japan Radiological Society, the Japanese Society of Hepato-BiliaryPancreatic Surgery, and the Japan Primary Care Association. This article has been co-published in the Japanese Journal of Radiology, Journal of Hepato-Biliary-Pancreatic Sciences, and Journal of General and Family Medicine. This research has been supported solely by these societies. All authors have reviewed the paper and agree with the contents.

Permission: Multiple copies, modification, alteration, enhancement, and/or distribution of this document are not permitted without the express permission of the Japan Radiological Society.

The affiliations of the members are as of October 2015.

Electronic supplementary material The online version of this article (doi:10.1007/s11604-015-0489-z) contains supplementary material, which is available to authorized users.

Toshihiko Mayumi

mtoshi@med.uoeh-u.ac.jp

1 Department of Emergency Medicine, School of Medicine, University of Occupational and Environmental Health, Kita-Kyushu, Japan
(CQs) and recommendations are shown in supplementary information].

Methods A systematic and comprehensive evaluation of the evidence for epidemiology, diagnosis, differential diagnosis, and primary treatment for acute abdomen was performed to develop the Practice Guidelines for Primary Care of Acute Abdomen 2015. Because many types of pathophysiological events underlie acute abdomen, these guidelines cover the primary care of adult patients with nontraumatic acute abdomen.

Results A total of 108 questions based on 9 subject areas were used to compile 113 recommendations. The subject areas included definition, epidemiology, history taking, physical examination, laboratory test, imaging studies, differential diagnosis, initial treatment, and education. Japanese medical circumstances were considered for grading the recommendations to assure useful information. The two-step methods for the initial management of acute abdomen were proposed. Early use of transfusion and analgesia, particularly intravenous acetaminophen, were recommended.

Conclusions The Practice Guidelines for Primary Care of Acute Abdomen 2015 have been prepared as the first evidence-based guidelines for the management of acute abdomen. We hope that these guidelines contribute to clinical practice and improve the primary care and prognosis of patients with acute abdomen.

2 Department of Hemodialysis and Surgery, Chemotherapy Research Institute, International University of Health and Welfare, Ichikawa, Japan

3 Department of General Internal Medicine, Hiroshima University Hospital, Hiroshima, Japan 
Keywords Acute abdomen - Guidelines · Two-step method $\cdot$ Diagnosis $\cdot$ Differential diagnosis $\cdot$ Analgesia

\section{Introduction}

Acute abdomen is any acute abdominal condition requiring a quick response. However, the abdominal discomfort associated with extra-abdominal pathophysiology and thoracic conditions such as acute myocardial infarction and pneumonia can at times mimic acute abdomen. The acute abdomen is managed by many kinds of physicians and requires accurate diagnosis and treatment within a particular time limit to prevent mortality. Furthermore, there was not enough high-quality evidence for the management of acute abdomen, and the management of acute abdomen depends on instinct and experience of the physicians. However, to date, clinical guidelines for physicians to facilitate best outcomes in patients with acute abdomen are lacking. In addition, methods of physical examination are changing with recent advances in diagnostic confirmation and treatment with modalities such as multidetector computed tomography. Acute abdomen is managed by many kinds of medical specialists. Therefore, the Japanese Society for Abdominal Emergency Medicine in collaboration with four other medical societies (Japan Radiological Society, Japan Primary Care Association, Japan Society of Obstetrics and Gynecology, Japanese Society for Vascular Surgery) initiated the preparation of Practice Guidelines for Primary Care of Acute Abdomen in 2012. After critical appraisal of evidence, hard discussion to formulate consensus and voting

4 Department of Radiological Sciences, Faculty of Health Sciences and Graduate School of Human Health Sciences, Tokyo Metropolitan University, Tokyo, Japan

5 Department of Obstetrics and Gynecology, University Hospital Mizonokuchi, Teikyo University School of Medicine, Kanagawa, Japan

6 Department of Vascular Surgery, International University of Health and Welfare, Mita Hospital, Tokyo, Japan

7 Division of Emergency and Critical Care Medicine, Department of Acute Medicine, Nihon University School of Medicine, Tokyo, Japan

8 Department of Obstetrics \& Gynecology, Juntendo University, School of Medicine, Tokyo, Japan

9 Department of Radiology, The Aichi Prefectural Federation of Agricultural Cooperatives for Health and Welfare Kainan Hospital, Aichi, Japan

10 Department of Radiology, Teikyo University School of Medicine, Tokyo, Japan

11 Emergency Department, Fukui Prefectural Hospital, Fukui, Japan

12 Center for Medical Education, University of Toyama, Toyama, Japan to make recommendations, the Practice Guidelines for Primary Care of Acute Abdomen 2015 that were the first English guidelines in the world for the management of acute abdomen were finally published.

\section{Methods}

\subsection{Purpose}

The purpose of these guidelines is to provide practical medical guidelines to clinicians for improving the quality and efficacy of treatment and advancing the prognosis and quality of life of patients with acute abdomen. However, the subjects of these guidelines are limited to adults with nontraumatic acute abdomen.

\subsection{Stakeholder involvement}

Members of the Committee of Japanese (JPN) Guidelines for Acute Abdomen 2015 included primary care physicians, gastroenterologists, surgeons, emergency physicians, radiologists, vascular surgeons, obstetricians, and gynecologists. The guidelines were first presented to related academic societies for public comments and were subsequently evaluated by a wide range of external parties, including the general public, attorneys, primary care and internal medicine physicians, and surgeons. These guidelines are designed for all clinicians who treat acute abdomen, ranging from general clinicians to the ones specializing in acute abdomen.

13 Department of Surgery, Surgical Oncology and Science, Sapporo Medical University School of Medicine, Sapporo, Japan

14 Department of Surgery, Keio University School of Medicine, Tokyo, Japan

15 Department of Primary Care and Emergency Medicine, Kyoto University Hospital, Kyoto, Japan

16 Department of Surgery, Ogaki Municipal Hospital, Ogaki, Japan

17 Comprehensive Internal Medicine, Shiga University of Medical Science, Shiga, Japan

18 Japan Society of Obstetrics and Gynecology, Tokyo, Japan

19 Japanese Society for Vascular Surgery, Tokyo, Japan

20 Japan Primary Care Association, Tokyo, Japan

21 Japan Radiological Society, Tokyo, Japan

22 Japanese Society for Abdominal Emergency Medicine, Tokyo, Japan 


\subsection{Guideline preparation method}

\subsubsection{Clinical question (CQ) preparation and literature search}

Members of the Committee of JPN Guidelines for Acute Abdomen 2015 discussed and determined CQs based on the important clinical issues listed. Keywords extracted from the CQ were used to retrieve academic articles published from January 1983 to December 2011 by querying PubMed and Japana Centra Revuo Medicina Web.

\subsubsection{Method of systematic literature review}

Evidence assessment was performed following the procedures described below.

1. Extraction of risk/benefit outcomes using CQs

2. Evaluation of each article: preparation of structured abstracts

The information in each article was summarized, including the study design, and the risk of bias in each study was determined.

3. Method of defining the quality of evidence supporting recommendations

Each study was evaluated using the Oxford Centers for Evidence-based Medicine 2011 Levels (Table 1) [1], and a comprehensive evaluation of the evidence was conducted for all articles indicated in CQs as body of evidence [2-17].

\subsection{Grading the strength of recommendations}

The strength of recommendations was graded with reference to (1) quality of the evidence, (2) preferences of the patient, (3) risks and benefits, (4) cost estimates, etc. The grading of recommendations was divided into 5 categories (Table 2) [18].

\section{Results}

One hundred and eight questions were prepared in nine subject areas, for which 113 recommendations were proposed (Tables 3, 4, 5, 6, 7, 8, 9, 10). All CQs and recommendations were shown in the Supplementary Information.

\subsection{Definitions (Table 3)}

CQ1 What is acute abdomen?

Acute abdomen refers to intra-abdominal pathology, including extra-abdominal, thoracic and systematic pathology, with an onset of less than one week that may require urgent interventions, such as surgery.

$<$ Comment $>$ Abdominal disease with acute onset of abdominal pain requiring immediate intervention including emergency surgery is termed acute abdomen. Abdominal pain is often derived from digestive system diseases but may also be caused by extra-abdominal diseases. Therefore, appropriate primary care should be provided based on careful history taking and local and systemic findings.

As urgent correspondence may be required without a definitive diagnosis in a proportion of cases, the general term 'acute abdomen' is used [19]. Abdominal pain is reported to be the chief complaint of as many as $5 \%$ of patients attending emergency rooms [20] (level 4). With consideration of the mechanisms and clinical pathophysiology underlying abdominal pain, aggravation should be avoided through immediate and adequate initial interventions including emergency surgery.

The causes of acute abdomen are classified into (1) localized abdominal pain; (2) clinical conditions, such as inflammation and/or infection, mechanical obstruction, circulatory disturbance; (3) extra-abdominal diseases; and (4) degree of emergency [21] (level 4).

\subsection{Epidemiology (Table 3)}

\section{CQ2 What is the incidence of acute abdomen?}

Causes of acute abdomen include acute appendicitis, cholelithiasis, small intestinal obstruction, ureteral stone, gastritis, peptic ulcer perforation, gastroenteritis, acute pancreatitis, diverticulitis, obstetrics and gynecological diseases; however, the incidence varies according to age and disease etiology (level 3). Furthermore, acute abdomen should be differentiated from myocardial infarction, torsion of the spermatic cord, and other systemic diseases.

CQ4 What are the prognostic factors of acute abdomen?

When acute abdomen is diagnosed to be due to cardiovascular diseases, or acute abdomen causes poor overall status and abnormal vital signs in patients, the prognosis is generally poor. The presence of comorbidities and advanced age are also known poor prognostic factors (level 2).

$<$ Comment $>$ High mortality and morbidity rates are observed in cases of acute abdomen due to cardiovascular diseases (myocardial infarction, mesenteric arterial occlusion, non-obstructive mesenterium ischemia, and aortic aneurysm rupture) [22] (level 2). Acute abdomen with abnormal vital signs (e.g., perforative large bowel peritonitis) and intestinal necrosis due to ileal strangulation have also been reported as poor prognostic factors [23] (level 3). 


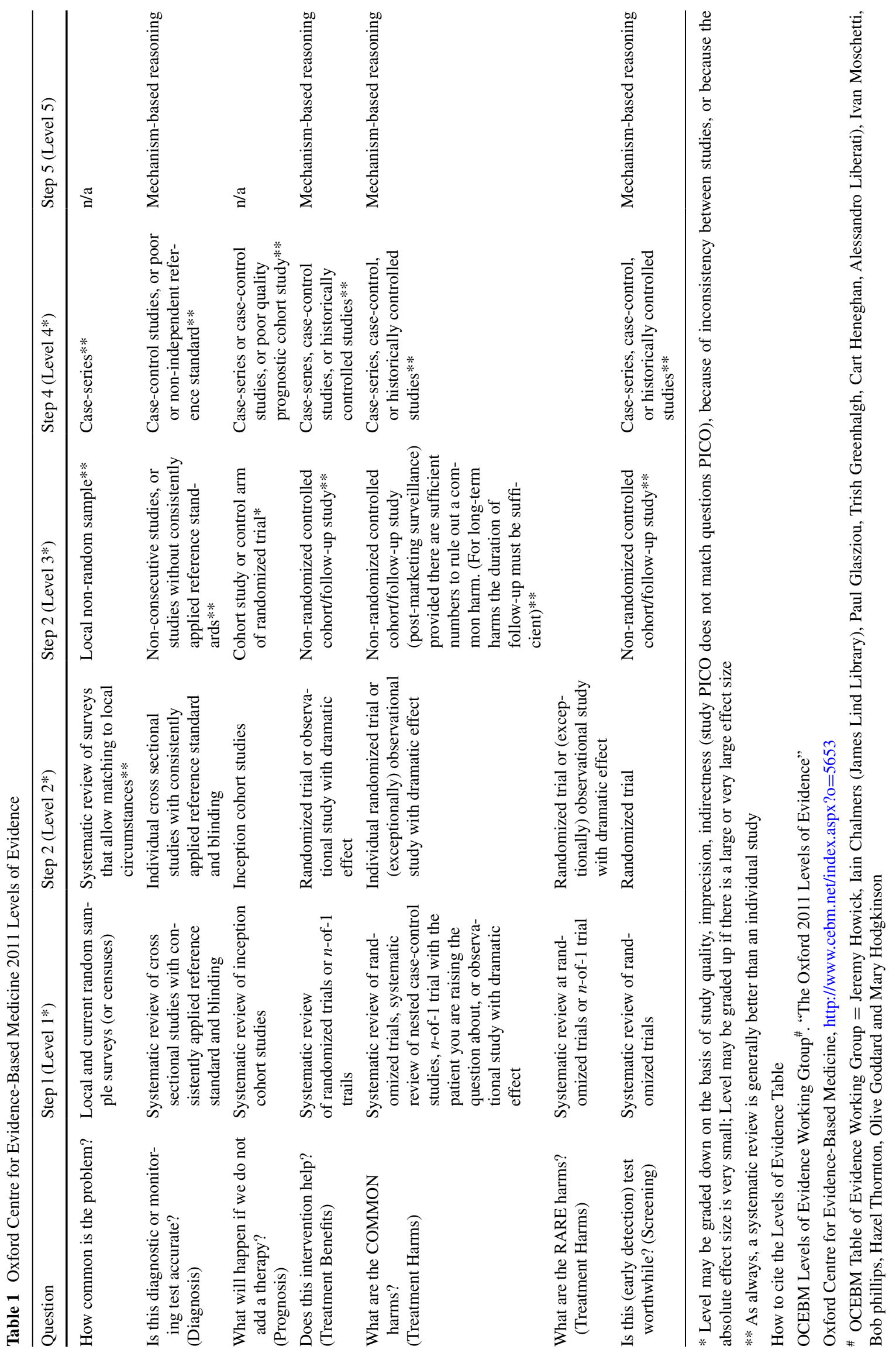


Table 2 Grades of recommendation $[\mathrm{J}$ Hepatobiliary Pancreat Sci (2010) 17:3-12]

\begin{tabular}{|c|c|}
\hline Grade of recommendation & Contents \\
\hline \multirow[t]{2}{*}{ A } & Recommended strongly to perform \\
\hline & Evidence is strong and clear clinical effectiveness can be expected \\
\hline \multirow[t]{2}{*}{ B } & Recommended to perform \\
\hline & Evidence is moderate or strong, although evidence of effectiveness is sparse \\
\hline \multirow[t]{2}{*}{$\mathrm{C} 1$} & Evidence is sparse, but may be considered to perform \\
\hline & Effectiveness can possibly be expected \\
\hline \multirow[t]{2}{*}{$\mathrm{C} 2$} & Scientific evidence is not sufficient, so clear recommendation cannot be made \\
\hline & Evidence is not sufficient to support or deny effectiveness \\
\hline \multirow[t]{2}{*}{$\mathrm{D}$} & Considered to be unacceptable \\
\hline & There is evidence to deny effectiveness (to show harm) \\
\hline
\end{tabular}

Table 3 Summary of recommendation: A. Definition, B. Epidemiology

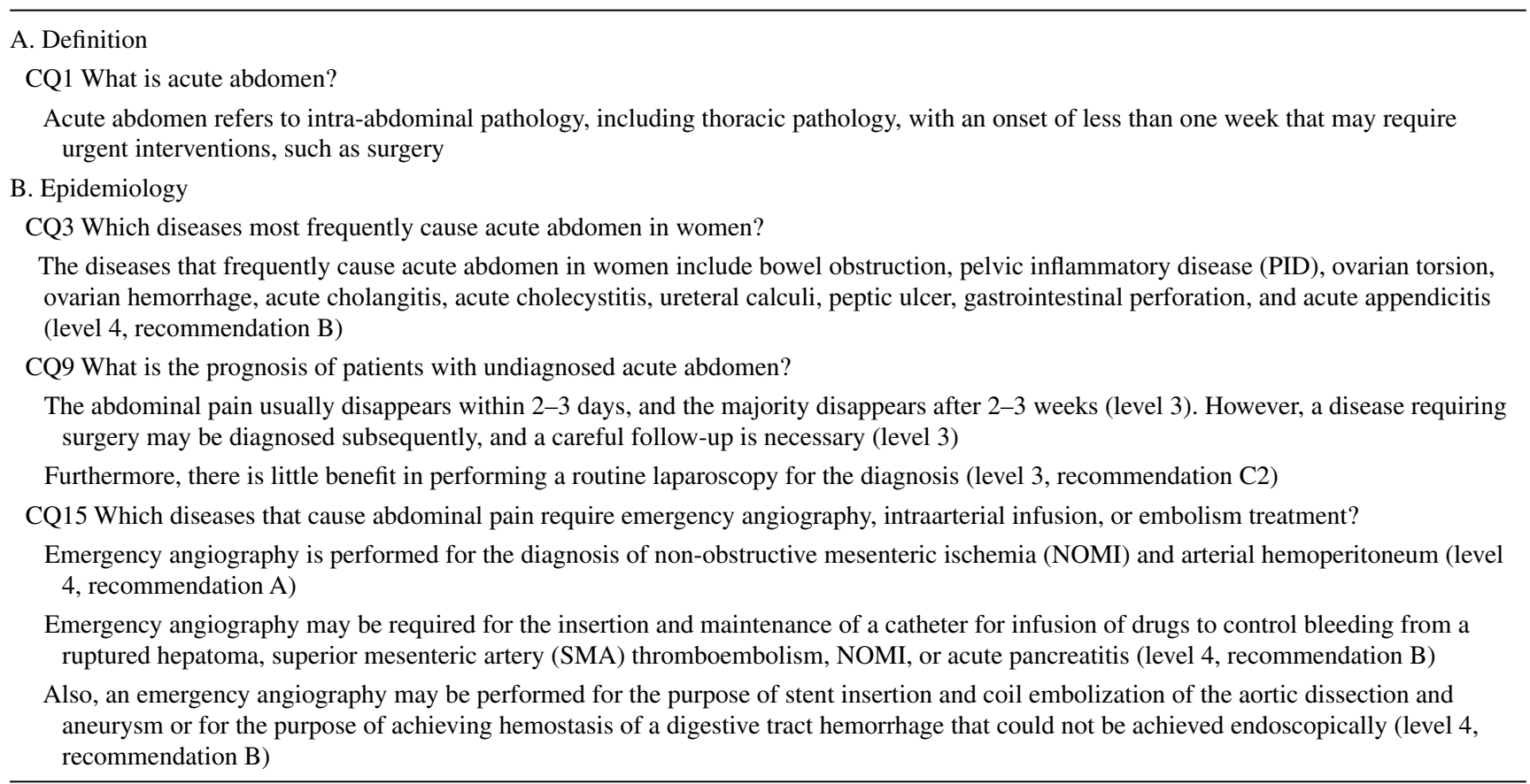

Data from National Clinical Database in 2011-2012 demonstrated that postoperative 30-day and in-hospital mortality rates in patients with general peritonitis were 8.8 and $14.1 \%$, respectively [24] (level 2).

High mortality and morbidity have been shown to be associated with poor general condition in elderly patients [25] (level 2) [26, 27] (level 3); steroid use [23] (level 3); respiratory or circulatory dysfunction; high APACHE II, SOFA, POSSUM, or E-PASS scores [28] (level 3); ASA classification class $\geq 3$ [23, 29-31] (level 3); organ failure [26, 32] (level 3); and recent invasive surgery [33] (level $3)$. Because the prevalence of cardiovascular comorbidities (myocardial infarction, mesenteric arterial occlusion, non-obstructive mesenterium ischemia, and aortic aneurysm rupture) and cardiopulmonary dysfunction is high in elderly patients, and diagnosis is usually more challenging in this population than in other generations, higher mortality from acute abdomen is observed in elderly patients [34] (level 3).

High volume centers typically have better acute abdomen outcomes than low volume centers [35] (level 2).

\subsection{History taking (Table 4)}

CQ16 What should be included in acute abdomen history taking?

The site and characteristics of the abdominal pain, accessory symptoms (pain location, migration, sudden onset, increasing severity, accompaniment with hematemesis/ hematochezia, vomiting, diarrhea, or constipation) should 
Table 4 Summary of recommendation: C. History taking

CQ16 What should be included in acute abdomen history taking?

The site and characteristics of the abdominal pain, accessory symptoms (pain location, migration, sudden onset, increasing severity, accompaniment with hematemesis/hematochezia, vomiting, diarrhea, or constipation) should be assessed to differentiate cases requiring emergency surgery (level 2, recommendation A)

Patient allergies, medication history, previous history of abdominal disease, diet, and pregnancy in women of reproductive age should be assessed (level 2, recommendation A)

CQ17 What medical and surgical history should be taken for patients with abdominal pain?

A complete medical and surgical history should be taken for patients with abdominal pain (level 5, recommendation A)

For patients with a history of ureteral calculi, cholecystolithiasis, or gastroduodenal ulcer, there are many recurring cases. Therefore, even if a complete medical history cannot be obtained, ascertain whether the patient had a similar pain previously (level 3, recommendation A)

Furthermore, even a minor abdominal surgery may cause adhesions and a strangulated bowel obstruction or an abdominal incisional hernia; therefore, a complete surgical history should be taken (level 5, recommendation A)

CQ18 Does taking a patient's history based on the SAMPLE contribute to treatment strategy decisions?

No clear evidence exists, showing that taking a history based on the SAMPLE contributes to treatment strategy decisions. However, the key questions based on the SAMPLE have become the gold standard for history taking during emergency care in many countries and are appropriate, particularly when time is limited (level 3, recommendation B)

CQ19 Is the general rule that otherwise healthy patients with intense abdominal pain continuing for more than $6 \mathrm{~h}$ more likely to undergo surgery?

There is no evidence that $6 \mathrm{~h}$ of continuous abdominal pain is valid grounds for surgery. However, because it is reported that continuous abdominal pain in elderly people is a predictor of emergency surgery, it is useful to check the duration of the abdominal pain (level 4 , recommendation $\mathrm{B}$ )

CQ20 What should be included in the medical history of patients with acute abdomen?

Patients with abdominal pain should be asked about all current medications (level 2, recommendation A)

CQ21 Does a patient's menstrual history (including the menstrual cycle and dysmenorrhea) have diagnostic significance?

Because differentiation of gynecological causes of acute abdomen, such as ovarian hemorrhage, endometriosis, or painful functional menses, and pregnancy-related causes of acute abdomen, such as extrauterine pregnancy or miscarriage, it is important that a menstrual history should be taken (level 4, recommendation B)

CQ22 Which questions are useful for determining pregnancy?

1. Has there been a delay in the patient's menstrual cycle?

2. Has the patient experienced any pregnancy symptoms, such as vomiting (morning sickness)?

3. Does the patient use contraception?

4. Has the patient had an opportunity to become pregnant?

5. Has the patient undergone infertility treatment?

These are the important questions to ask to determine pregnancy (level 1, recommendation A). However, pregnancy may be overlooked even if the answers are not indicative of pregnancy. When it is necessary to definitively diagnose pregnancy, urinary or serum hCG should be measured

CQ23 Is vomiting accompanying the abdominal pain useful for a diagnosis?

Vomiting in conjunction with acute abdomen is an important sign of the following diseases and conditions:

1. Excessive stimulation of the peritoneal or mesenteric nerve, gastric ulcer perforation, acute pancreatitis, appendicitis perforation, and ovarian torsion

2. Obstruction of the lumen of an organ consisting of involuntary muscles (the bile duct, the urinary tract, the cervical canal, the bowel, and the appendix)

The presence of vomiting and characteristics of the vomit should be checked (level 5, recommendation A)

CQ25 Is anorexia coexisting with abdominal pain useful for the diagnosis?

Anorexia is a nonspecific symptom accompanying many diseases. When acute appendicitis is suspected, the presence of anorexia should be confirmed (level 3, recommendation C1)

CQ26 Is a change in bowel habits with abdominal pain useful for a diagnosis?

A change in bowel habits may accompany digestive system diseases, such as colorectal cancer. The probability is particularly high in patients under 70 years of age. Bowel habits should be ascertained for patients with acute abdomen (level 3, recommendation A)

CQ27 Are the presence and properties of constipation and/or diarrhea useful for the diagnosis of acute abdomen?

The presence of and the number of times a patient has experienced diarrhea and/or constipation are useful in the diagnosis of acute abdomen and may be a symptom of bowel obstruction. The presence and characteristics of the diarrhea and/or constipation should be ascertained (level 4, recommendation A) 
Table 4 continued

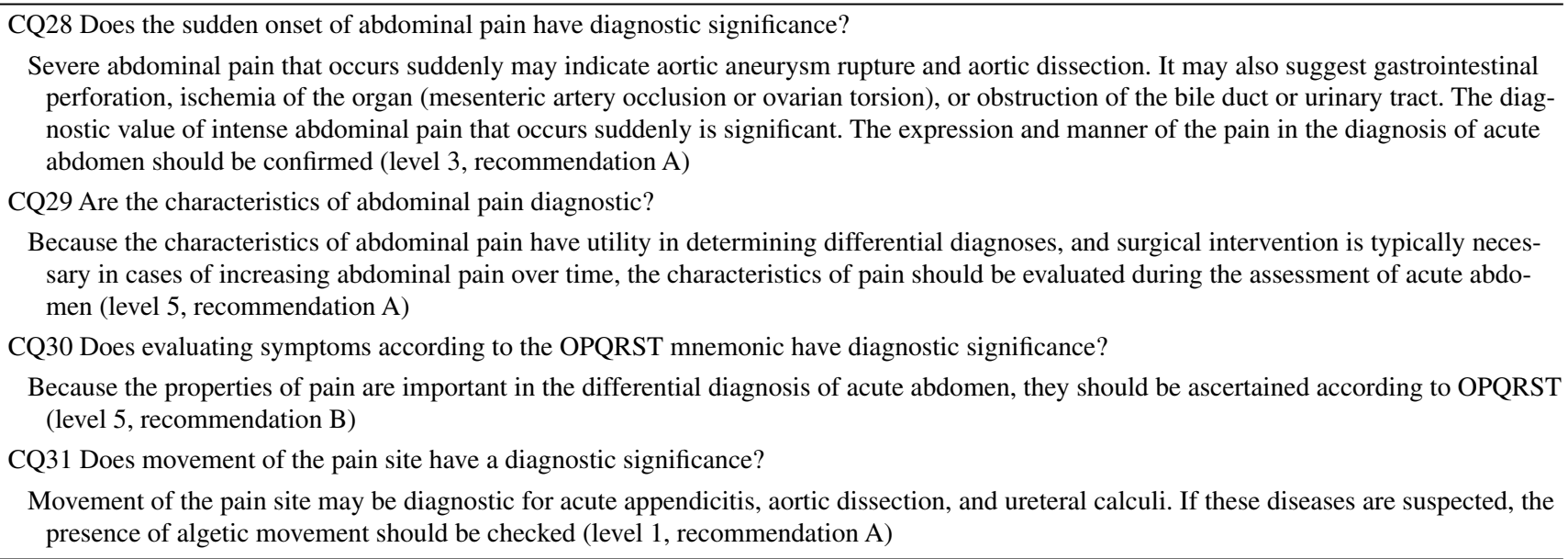

be assessed to differentiate cases requiring emergency surgery (level 2, recommendation A).

Patient allergies, medication history, previous history of abdominal disease, diet, and pregnancy in women of reproductive age should be assessed (level 2, recommendation A).

$<$ Comment> In patients with intense pain and unstable vital signs, sufficient time may not be available for careful taking of complete history. In these cases, a brief medical history should be taken to differentiate cases requiring emergency treatment (Fig. 1) (level 4). The acronym "SAMPLE" has been proposed as a method of obtaining useful information in a timely manner [36] (level 4). In addition, the acronym "OPQRST" [O (onset), $\mathrm{P}$ (palliative/ provocative), Q (quality/quantity), R (region/radiation), $\mathrm{S}$ (associated symptom), $\mathrm{T}$ (time course)] has also been recommended as a systematic and comprehensive method of medical history taking [37] (level 4).

Pain location is often useful in determining differential diagnoses (Fig. 2). For example, right upper quadrant pain is often observed in acute cholecystitis and hepatitis, whereas pain migrating from the upper quadrant to the right lower quadrant may indicate acute appendicitis. On the other hand, diverticulitis usually produces pain in the central, right, or left abdominal regions. Esophagitis and peptic ulcers may cause epigastric discomfort.

Pain radiation is another important component of the abdominal examination. Pain derived from pancreatitis usually spreads through the back, and pain from renal colic typically radiates towards the reproductive organs [38] (level 4).

Pain duration is important in cases of both acute and intermittent abdominal pain. Pain from pancreatitis typically increases gradually and persists. On the other hand, pain from perforated peritonitis has a rapid onset and is severe from the time of onset [38] (level 4).
The characteristics of reported pain may also assist in diagnosis. Typical reflux esophagitis causes burning pain, whereas gastroenteritis and bowel obstruction may cause colicky pain [38] (level 4).

In general, reported pain severity is correlated with disease severity. This is particularly true of acute onset abdominal pain. For example, colic due to gallstones, renal calculus, and mesenteric artery occlusion is typically very severe, whereas pain due to gastroenteritis is less intense. However, differentiation is often difficult as pain thresholds are subjective and vary according to the character and pain experience of the patient. Furthermore, patient age and daily health status may influence the perception of pain. Pain is commonly masked in patients taking steroids and complaints of pain are often obscure in elderly patients. A comparison with previously experienced pain is often useful [38] (level 4).

The identification of factors that exacerbate or reduce pain is also important. Pain from peptic ulcer is relieved by eating and then develops again after several hours. Pain from pancreatitis is typically relieved by sitting and worsens with decubitus [38] (level 4).

The determination of symptoms associated with abdominal pain is important. In patients with vomiting or diarrhea and abdominal pain, viral enteritis (e.g., norovirus), bacterial enteritis (e.g., Campylobacter), and acute gastric mucosal lesion due to Anisakis should be considered. Weight loss is often observed in patients with malignant tumors. Sustained nausea and/or vomiting indicates bowel obstruction. Change in bowel habit may indicate large bowel disease. [38] (level 4).

Pregnancy should be excluded in women of childbearing age. The period and duration of last menstruation, menstrual cycle, irregular bleeding, contraceptive use, and history of sterility treatment should be obtained [38] (level 4).

Peptic ulcers associated with nonsteroidal anti-inflammatory drug (NSAID) use, and pseudomembranous 
Table 5 Summary of recommendation: D. Physical Examination

CQ32 What is the most appropriate physical examination method when acute abdomen is suspected?

The evaluation of patient appearance, vital signs, and an estimate pain degree/severity should be performed to assess the requirement for emergency surgery

The abdominal medical examination comprises ocular inspection, auscultation, percussion, and palpation

The chest, lower back, rectum, and urogenital apparatus should be examined as required (level 5, recommendation A)

CQ33 Does a clinician's first impression of a patient have utility in the diagnosis of acute abdomen?

First impression (expression, complexion, respiratory status, conditioning, manner, etc.) provides information regarding the pain site and peritoneal stimulation sign. This information may be used to evaluate the urgency and severity of abdominal pain (level 3, recommendation B)

CQ35 Does the measurement of vital signs have utility in patients with acute abdomen?

Tachypnea raises the probability of pneumonia, cardiorespiratory failure, and bacteremia (level 2)

Tachycardia, hypotension, and body temperature are correlated with severity and prognosis (level 3 )

Therefore, vital signs should always be measured in patients with acute abdomen (recommendation A)

CQ36 Is an abdominal ocular inspection useful in the diagnosis of acute abdomen?

An abdominal ocular inspection should be performed to check for surgery scars, skin findings, abdominal distension (local or whole?), hernia, abdominal beat, an abdominal mass, and abdominal wall exercise during breathing (level 2, recommendation B)

Abdominal distension, intestinal peristalsis, and a surgery scar detected during the ocular inspection of patients with acute abdomen very likely indicate a bowel obstruction (level 4, recommendation B)

CQ37 Should auscultation for multiple intestinal peristalsis be performed at a particular location?

Although auscultation is a mandatory component of the abdominal medical examination, there is a lack of consensus regarding the standard method of abdominal auscultation

If the conduction of peristaltic sounds is good, auscultation at a single location is considered adequate (level 4, recommendation C1)

Because the clinical significance of auscultation is limited in patients with acute abdomen, in whom peristaltic sounds cannot be heard, it is not recommended that multiple auscultations be performed at different locations or for increased durations (level 5, recommendation C2)

CQ38 Does abdominal examination using a stethoscope have utility in the diagnosis of acute abdomen?

The identification of abnormal peristaltic murmurs has utility in the diagnosis of bowel obstruction (level 3, recommendation B)

The significance of abdominal bruits is currently unknown in cases of acute abdomen (level 5, recommendation C2)

CQ39 Does abdominal percussion have utility in the diagnosis of acute abdomen?

The presence or absence of percussion pain (percussion tenderness) and ascites can be detected by abdominal percussion in cases of acute abdomen (level 2, recommendation A)

CQ40 Does abdominal palpation have utility in the diagnosis of acute abdomen?

Muscle guarding, muscle rigidity, and rebound tenderness on light palpation are required to confirm the presence of a peritoneal stimulation sign (CQ42, 43) (level 2, recommendation A)

Organ enlargement or abdominal cavity masses such as those in the gallbladder, liver, spleen, or bladder may be detected on deep palpation (level 2, recommendation $\mathrm{C} 1$ )

CQ41 Are the ascitic findings, the iliopsoas muscle test, the obturator test, and the Howship-Romberg sign useful in the diagnosis of acute abdomen?

In the diagnosis of acute abdomen, the examination procedure for ascites is not important (level 5, recommendation C2)

When acute appendicitis is suspected, the iliopsoas muscle test and the obturator test are recommended (level 3, recommendation C1)

When an obturator hernia is suspected, the Howship-Romberg sign should be confirmed (level 4, recommendation C1)

CQ43 Does peritoneal stimulation have utility in the diagnosis of acute abdomen?

The peritoneal stimulation sign suggests peritonitis (level 2, recommendation B)

If percussion pain is positive, it does not necessarily induce rebound tenderness (level 3, recommendation C2)

Imaging studies are recommended when peritonitis or mesenteric vascular disorder are suspected clinically (level 5, recommendation B)

CQ44 How does obesity index affect the diagnosis of acute abdomen?

The obesity index does not affect the diagnosis of abdominal pain in Japan (level 4). However, a physical examination may become difficult as the obesity index increases, and thus, imaging studies should be added (level 5, recommendation $\mathrm{C} 1$ )

CQ45 Does the abdominal wall tenderness test (Carnett's sign) have utility in the diagnosis of acute abdomen?

The abdominal wall tenderness test has utility in diagnosing abdominal wall ache or psychogenic stomachache and excluding intra-abdominal lesions (level 4, recommendation $\mathrm{C} 1$ )

CQ46 Is a rectal examination useful for the diagnosis of acute abdomen?

Because the information obtained by adding a rectal examination is extremely limited, and a rectal examination is sometimes distressing for the patient, it is not recommended for the routine examination in the diagnosis of the acute abdomen (level 3, recommendation C2) 
Table 5 continued

However, a rectal examination may be indicated when it is necessary to examine the stool properties and when anal diseases, such as hemorrhoids and anal fistula, digestive tract hemorrhage, rectal cancer, prostate cancer, or prostatitis, are suspected (level 3, recommendation B)

CQ47 Is a vaginal examination useful in the diagnosis of acute abdomen?

There is no definitive evidence showing the utility of a routine internal examination of women with acute abdomen in the emergency room. When extrauterine pregnancy or gynecologic pathology, including pelvic inflammatory disease (PID), is suspected, pain upon movement of the cervical canal and appendicular tenderness on internal examination may be useful in the diagnosis (level 2, recommendation C1)

Table 6 Summary of recommendation: E. Laboratory Test

CQ50 Does the measurement of procalcitonin (PCT) have utility in the diagnosis and evaluation of acute abdomen severity?

The utility of PCT in the diagnosis of non-perforated appendicitis has not been shown to be greater than that of CRP or white blood cell (WBC) count; however, PCT has utility in the diagnosis of perforative and abscess formation-related appendicitis. PCT has greater utility in the evaluation of acute abdomen severity of acute pancreatitis and peritonitis (level 2, recommendation B)

CQ51 In which etiology of acute abdomen does blood gas analysis (including lactate) have utility?

The measurement of base excess (BE), pH, and serum lactic acid level has utility in the diagnosis of shock and intestinal ischemia, and it is commonly performed in patients with high levels of clinical suspicion (level 2, recommendation B)

However, serum lactate levels may not be increased in cases of early intestinal ischemia or volvulus

CQ52 When the white blood cell count and CRP are normal, is appendicitis excluded?

It is reported that the white blood cell count is normal in approximately $40 \%$ of appendicitis cases, and CRP may be normal without perforation or abscess formation. Therefore, a normal white blood cell count and CRP level should not exclude a diagnosis of appendicitis (level 2 , recommendation D)

CQ53 Is it useful in the differential diagnosis to measure lipase and amylase in patients with epigastric pain?

Measurement of lipase is particularly useful in the diagnosis of the acute pancreatitis but is of little value for diagnosing other diseases (level 3 , recommendation $\mathrm{B}$ )

CQ54 For which patients with acute abdominal pain should an electrocardiogram be recorded?

Because patients with epigastric pain who also have the risk factors of ischemic heart disease may have a myocardial infarction, an electrocardiogram is necessary (level 2, recommendation A). Also, it is necessary to confirm the presence or absence of atrial fibrillation, when mesenteric, splenic, or renal ischemia or infarction is suspected (level 3, recommendation B)

CQ56 In which patients do urinalysis have utility?

Measurement of urinary human chorionic gonadotropin (hCG) levels has utility in the diagnosis of pregnancy. Measurement of urinary porphobilinogen has utility in the diagnosis of acute porphyria. Urine qualitative analysis has utility in the diagnosis of ureteral stones, urinary tract infection, and ketoacidosis (level 3, recommendation B)

CQ57 Can ureteral calculi be confirmed or excluded by occult blood in the urine?

The sensitivity and false-positive rate of urine occult blood in patients with ureteral calculi are 20-30\%. Therefore, it is impossible to diagnose or exclude ureteral calculi only by occult blood in the urine, and it is desirable to perform imaging tests, such as ultrasonography, for confirmation (level 3, recommendation B)

CQ58 For which patients with abdominal pain is a pregnancy test useful?

Pregnancy tests are recommended for female patients of childbearing age who cannot rule out pregnancy (patients with sexual experience before the menopause and after the menarche and patients undergoing infertility treatment) (level 3, recommendation B)

CQ59 For which patients with abdominal pain is an examination for sexually transmitted diseases useful?

It is useful in the female patient with the sexual experience, particularly patients with suspected pelvic inflammatory disease (PID) (level 3, recommendation $\mathrm{C} 1$ )

enterocolitis due to antimicrobial agents should be considered.

In patients with previous history of surgery, detailed surgical history is recommended to assess the possibility of adhesive and strangulated bowel obstruction [38] (level 4).

Because recurrence of ureteral stones, cholelithiasis, and gastroduodenal ulcers is common, the identification of any previous history of these diseases is important [38] (level 4).
CQ20 What should be included in the medical history of patients with acute abdomen?

Patients with abdominal pain should be asked about all current medications (level 2, recommendation A).

$<$ Comment $>$ Because a large range of medications may be associated with acute abdomen, all currently medications should be determined [38] (level 4). In particular, the 
Table 7 Summary of recommendation: F. Imaging Test

CQ60 In which cases of acute abdomen should plain abdominal radiography be performed?

The diagnosability of plain abdominal radiography is limited and has no value as a part of routine examinations. Even in cases without abnormal findings, ultrasonography (US) or computed tomography (CT) should be considered depending on clinical manifestations (level 2, recommendation $\mathrm{C} 2$ )

However, in settings without access to US or CT, X-ray is considered in patients suspected to have bowel obstruction, ileus, gastrointestinal perforation, calculus urinary, emphysematous lesion, or a foreign body (level 2, recommendation $\mathrm{C} 1$ )

CQ61 What kind of scans during the course of abdominal radiography should be used for acute abdomen?

For the initial abdominal radiographic examination, dorsal and standing scans are common, and a chest standing position radiographic examination is often added (acute abdomen series*). When a standing position is difficult, a left lateral decubitus position can be substituted (level 5, recommendation B). However, for diseases other than bowel obstruction and ileus, the utility of the abdominal standing position radiograph may be low and therefore omitted (level 4, recommendation $\mathrm{C}$ )

* The acute abdomen series consists of a set of abdominal radiographs in the dorsal and standing position and a chest radiograph in the standing position

CQ62 In which cases of acute abdomen should plain chest radiography be considered?

When gastrointestinal perforation or diseases of the chest such as pneumonia, pericarditis, or myocardial infarction are suspected, plain chest radiography should be considered (level 3, recommendation B)

CQ63 For which kind of acute abdomen case is a contrast radiographic study useful?

For esophageal and gastric perforations, an upper GI series using water-soluble iodinated contrast media* is useful. When there is a risk of aspiration, a low- or isosmotic contrast medium is recommended (level 4, recommendation B). Also, an oral contrast study (water-soluble contrast media) for small intestinal obstruction and a radiographic contrast enema for large intestinal obstruction are useful (level 4). Antidromic cystography is useful for diagnosing urinary bladder rupture (level 3)

* Each water-soluble iodinated contrast medium has a tri-iodinated benzene ring in the fabric. Some ionic contrast media contain side chains that keep solutions ionized, whereas the nonionic contrast media do not ionize. The former is more hypertonic than the latter because the osmotic pressure of the ionic contrast media is as high as 9 times that of the plasma (75\% of the iodine concentration) and is therefore called hypertonic contrast media. However, because the osmotic pressure of a nonionic contrast medium is as low as 3 times that of the plasma (60\% of the iodine concentration), it is called a low-osmolar contrast medium. The osmotic pressure of the iso-osmolar contrast media is even lower, approximately equivalent to plasma osmolality. Because serious pneumonia and pulmonary edema may develop when hypertonic contrast media is in the bronchus, use of low- or iso-osmotic contrast media is recommended when the aspiration of contrast media might occur (level 4)

CQ64 What disease should be assumed when radiographs shows calcification?

Acute abdomen with calcification includes ureteral calculi, acute appendicitis, acute cholecystitis, acute pancreatitis (acute aggravation of chronic pancreatitis), Meckel's diverticulitis, intestinal ischemia, aneurysm (impending) rupture, aortic dissection, tumor (rupture, twisting, or infarction), bowel obstruction, and bowel perforation (level 5). From the position, shape, and mobility of the calcification, it is important to judge what kind of calcification it is and its association with acute abdomen (level 5, recommendation B). However, calcification discovered by abdominal radiography may be unrelated to the cause of the acute abdomen (level 5)

CQ65 In which cases of acute abdomen is US required?

US is recommended as a screening test for acute abdomen and strongly recommended particularly when AAA rupture or acute cholecystitis is suspected. US is recommended in pregnant women, young woman, or children in whom radioactive exposure is not desirable (level 2 , recommendation A). However, expertise in US is required as this technique is highly dependent on practitioner skill (level 2, recommendation A)

CQ66 In which cases of acute abdomen should CT be performed?

CT may be indicated in all patients with acute abdomen (level 2, recommendation A). However, CT can be omitted when a diagnosis is made according to the results of precedent examinations such as US. Radiation exposure should be considered with the use of CT

CQ68 For which cases of acute abdomen are enhanced CT image useful?

Because a detailed evaluation of organ ischemia, vascular lesions, or acute pancreatitis severity may be difficult using only non-enhanced CT, enhanced CT is recommended. In most cases, diagnoses are made by enhanced CT only, but for the evaluation of blood circulation disorders due to strangulation ileus and for the diagnosis of thrombosed type aortic dissection, both plain and enhanced CT together are desirable (level 3, recommendation B)

CQ69 Which clinical conditions be considered when contrast media is administered for CT and MRI studies in patients with acute abdomen?

Contrast media such as iodine gadolinium should be administered in patients with a history of asthma and allergies only when the benefit sufficiently exceeds that of an equivalent test (level 5, recommendation C1). Furthermore, nephropathy induced by iodinated contrast media and the renal systemic fibrosis induced by gadolinium contrast media have been reported in patients with renal dysfunction, and careful attention must be paid to those patients in particular. In patients taking a biguanide for diabetes, attention must be paid to the potential onset of lactic acidosis after iodinated contrast media administration (level 5, recommendation $\mathrm{C} 1$ ) 
Table 7 continued

CQ70 In patients with abdominal pain and no abnormality detected on CT, which emergent diseases can be excluded?

CT has high diagnosability for intestinal ischemia, gastrointestinal perforation, acute appendicitis, diverticulitis, biliary tract calculus, and acute pancreatitis among others. However, these diseases cannot be completely ruled out, even if abnormalities are not found on CT (level

3 , recommendation $\mathrm{B}$ )

CQ71 What type of imaging study is useful for detecting intra-abdominal free air?

CT (if possible, multiple detector CT) is recommended (level 3, recommendation B)

CQ72 What is the role of abdominal US and CT in cases of peritonitis due to acute abdomen?

The diagnosis of peritonitis due to acute abdomen is possible from medical history, physical examination, and laboratory testing in the majority of cases; however, imaging studies should be performed to confirm peritonitis and the underlying cause of investigation

US is the initial imaging study of choice; however, enhanced abdominal or pelvis CT (when possible, plain + enhanced) should be performed in cases where a definitive diagnosis has not been reached with US (level 4, recommendation B)

CQ73 For what kind of acute abdomen case should MRI be performed?

MRI should be considered for acute abdomen due to hepatobiliary disease and gynecological disease for which a diagnosis is not obtained by ultrasonography and CT as well as for a pregnant woman for whom ultrasonography does not lead to a diagnosis (level 2, recommendation C1)

CQ75 What precautions should be taken for the MRI imaging of a pregnant woman?

There is no high-quality evidence contraindicating MRI during any period of pregnancy, but because its safety has not yet been completely established in early pregnancy, ultrasonography is recommended first. When a diagnosis is not obtained by ultrasonography, non-enhanced MRI may be considered (level 2, recommendation C1). Although the enhanced MRI is not contraindicated, it should be considered only when the benefit sufficiently exceeds that of the equivalent test (level 4 , recommendation $\mathrm{C} 2$ ).

type and quantity of NSAIDs and steroid therapies should be determined.

In a meta-analysis of 18 studies published in the 1990s, risk of bleeding or perforation of the upper gastrointestinal tract was observed to be increased by 3.8 times by the use of NSAIDs, and this increase was greater in elderly men [39] (level 1).

A retrospective study in New York in 1986 reported that the peritoneal irritation sign was infrequently observed in cases of intestinal perforation among patients receiving steroid therapy [40] (level 3). Another study in Minnesota reported fewer symptoms of fever, muscle guarding, and rigidity in patients with gastrointestinal perforation who were administered $>20 \mathrm{mg}$ /day prednisolone [41] (level 3). Therefore, symptoms may not always be apparent in patients receiving steroid therapy.

\section{CQ29 Are the characteristics of abdominal pain diag- nostic?}

Because the characteristics of abdominal pain have utility in determining differential diagnoses, and surgical intervention is typically necessary in cases of increasing abdominal pain over time, the characteristics of pain should be evaluated during the assessment of acute abdomen (level 5, recommendation A).

$<$ Comment $>$ The characteristics (pattern of manifestation, intensity, type, and duration) of the pain may often indicate the pathologic nature and severity of the reported pain [19] (level 5).
Types of abdominal pain are typically divided into colicky (visceral pain) and somatic pain. Colicky pain is derived from the twitching of the smooth muscle of luminal organs (alimentary canal, urinary tract, or uterus) and from rapid extension and expansion of the visceral peritoneum. Colicky pain is periodic, intermittent, and cramp-like in nature. Colic duration of 4-5 min may indicate jejunal obstruction, 8-10 min may indicate ileal obstruction, and of 15 min may indicate large bowel obstruction [19] (level 5). Furthermore, severe abdominal pain lasting more than $6 \mathrm{~h}$ has been reported as an indication for surgical intervention [19] (level 5).

On the other hand, somatic pain is persistent and prickling in nature, and it is derived from local inflammation due to stimulation of the parietal peritoneum and mesenterium.

\subsection{Physical examination (Table 5)}

CQ32 What is the most appropriate physical examination method when acute abdomen is suspected?

The evaluation of patient appearance, vital signs, and an estimate pain degree/severity should be performed to assess the requirement for emergency surgery.

The abdominal medical examination comprises ocular inspection, auscultation, percussion, and palpation.

The chest, lower back, rectum, and urogenital apparatus should be examined as required (level 5, recommendation A).

$<$ Comment $>$ 
Table 8 Summary of recommendation: F. Differential Diagnosis

CQ88 Which diseases should be differentiated for acute abdomen in a pregnant woman?

The following pregnancy-related conditions should be considered: extrauterine pregnancy, including simultaneous intrauterine and abdominal pregnancy, miscarriage, labor, premature separation of a normally implanted placenta, uterine rupture, round ligament pain, chorioamnionitis, and HELLP syndrome

The following non-pregnancy-related conditions should also be considered: appendicitis, cholecystitis, bowel obstruction, acute pancreatitis, urinary calculus, ovarian torsion, uterine myoma, and acute abdomen due to other nonspecific abdominal pain (level 4, recommendation C1)

CQ89 What are the characteristic, prognostic, and predictive features of acute abdomen in elderly patients?

Regarding the characteristics of acute abdomen in elderly patients, the results of abdominal physical examination and laboratory testing often do not reflect the patient condition, decisions regarding surgical indications and prognosis are challenging (level 3), and a definitive diagnosis is often not possible (level 3). As CT has utility in the diagnosis of acute abdomen and informing decisions regarding treatment strategies, abdominal CT is recommended as required (level 3, recommendation B)

Urgent hospitalization and surgical intervention are often required for elderly people complaining of abdominal pain (level 3)

Further, mortality rates increase with patient age

Acute intestinal ischemia, AAA rupture, and organ dysfunction of the extra-abdominal cavity are all associated with high mortality rates (level 3)

CQ90 What characteristics should be considered for acute abdomen in immunocompromised patients?

Even with severe peritonitis, physical examination findings in immunocompromised patients may be poor, and it is necessary to evaluate even minor findings carefully. For example, the patient's body temperature tends not to be elevated. Even if the clinical symptoms and signs are weak, laboratory tests and imaging should be used to evaluate the patients carefully (level 5, recommendation B)

CQ91 What characteristics should be considered for acute abdomen in patients with paralysis and sensory disturbance?

Abdominal pain may be hidden by sensory disturbance, and a delay in diagnosis can increase mortality. Exacerbation of the physical findings that are not affected by sensory disturbance must be considered, and evaluation using an objective index apart from the physical examination should also be considered (level 4, recommendation B)

CQ94 What should be considered in the patients for whom mutual understanding is difficult, such as dementia patients?

The patient's condition is apt to be judged to be mild because expression and evidence of abdominal pain may be poor, but mild findings should not be overlooked, and an imaging study, including a CT scan, is recommended (level 4, recommendation A). Regarding potential diseases, megacolon (perforative), acute appendicitis, gastrointestinal perforation, foreign body allotriophagy, and side effects of antipsychotics require attention (level 5)

CQ95 What information contributes to the diagnosis of idiopathic bacterial peritonitis?

Idiopathic bacterial peritonitis results from an underlying disease that produces ascites where cirrhosis is most common. In addition, it may be complicated by ascites occurring in conjunction with the nephrotic syndrome, a malignant tumor, acute hepatitis, or collagen disease

Idiopathic bacterial peritonitis is diagnosed by a neutrophil count in the ascitic fluid. Because the frequency of symptoms, such as fever, stomachache, abdominal tenderness, and rebound tenderness, is low, a neutrophil count in the ascites fluid is required for diagnosis (level 2 , recommendation $\mathrm{A}$ )

CQ96 When should ovarian torsion be suspected?

When an ovarian cyst is detected by an imaging study in patients with lower abdominal pain, an obstetrician should be consulted immediately. Ovarian torsion should be considered in patients with a history of a previous ovarian cyst or assisted reproductive technology, in pregnant women, and in women of childbearing age (level 3, recommendation A)

CQ97 When should an obstetrician be consulted for suspicion of extrauterine pregnancy?

Characteristic symptoms of extrauterine pregnancy with acute abdomen include amenorrhea, genital bleeding, and lower abdominal pain. When ultrasonography reveals no fetal sacs in the uterus after 6 weeks of pregnancy or when fetal sac-like structures and effusion liquid outside the uterine cavity are detected, an obstetrician should be consulted immediately (level 1, recommendation A)

CQ98 What are the physical, laboratory, and imaging findings that suggest pelvic inflammatory disease (PID)?

1. Physical examination findings suggesting PID include lower abdominal tenderness, rebound tenderness, muscle guarding, positive heel drop test, tenderness of the pelvic organs, and tenderness of the appendicular region and pain when the uterine cervix is moved by a bimanual examination (rectal examination) (level 4, recommendation $\mathrm{C} 1$ )

2. The laboratory finding suggesting PID is a remarkable increase in the CRP level (level 4, recommendation C1)

3. A transvaginal ultrasonography finding highly specific for PID is a bright ascitic fluid. Fallopian tubes ovarian or cyst that the contents bright ingredient for fallopian tubes or ovarian abscesses, also suggests PID (level 4, recommendation C1)

4. Generally, the findings of enhanced CT are nonspecific, but a low-absorbance mass and bowel edema in the pelvis with hyperplasia of the wall of the fallopian tubes and ovarian abscess are suggestive of PID (level 4, recommendation C1) 
Table 9 Summary of recommendation: G. Initial Therapy

CQ102 What is initial treatment when acute abdomen is suspected?

First, the patient's vital signs and airway (A), breathing (B), circulation (C), and consciousness, should be confirmed, and emergency treatment provided upon the determination of abnormal vital signs and $\mathrm{ABC}$ and treatment for the causative disease, if known, should be initiated immediately. When radical treatment is challenging, emergency treatment should be provided and hospital transfer should be considered (Fig. 1) (level 4, recommendation A)

When vital signs are stable, decisions regarding the need for emergency surgery should be made based on medical history and abdominal findings. In addition, laboratory tests and imaging studies have utility in determining whether the clinical condition (bleeding, organ ischemia, pan-peritonitis, acute inflammation) requires surgical intervention (Fig. 1) (level 4, recommendation A)

CQ103 How should initial transfusions be initiated in cases of acute abdomen?

Initial transfusions should be initiated immediately when intraperitoneal infections are diagnosed, even if circulatory dynamics are stable (level 3, recommendation A)

The stabilization circulatory dynamics has top priority in cases of shock (level 5, recommendation A)

Crystalloid solutions, such as Ringer's solutions, should be used (level 1, recommendation A)

Hydroxyethyl starch (HES) is not recommended (level 1, recommendation D)

The use of albumin preparation should be considered in patients in shock and requiring large transfusion volumes or who have hypoalbuminemia (level 1, recommendation $\mathrm{C} 1$ )

Erythrocyte transfusion should be provided with hemoglobin levels less than 7-9 g/dl (level 1, recommendation A)

CQ104 What is the preferable transfusion route in acute abdomen?

For initial fluid therapy, transfusion should be started using a peripheral vein (level 3, recommendation A). For a patient in shock, treatment should be provided according to early goal-directed therapy, and a central venous catheter should be inserted (level 3, recommendation B) When insertion via a peripheral intravenous route is difficult, a bone marrow transfusion method should be considered for both children and adults (level 3, recommendation B)

CQ105 Which analgesic should be used for abdominal pain due to acute abdomen?

Regardless of cause, early analgesic use before a definitive diagnosis is recommended

Intravenous administration of $1,000 \mathrm{mg}$ acetaminophen is recommended regardless of pain severity (level 1 , recommendation A)

Intravenous narcotic analgesics should be added according to pain severity

Anticonvulsants, such as butylscopolamine bromide, may be used as an adjuvant therapy for colic rather than the first drug of choice for abdominal pain (level 1, recommendation A)

Morphine, opioids such as fentanyl (level 1, recommendation A), an antagonistic analgesic such as pentazocine, and buprenorphine (level 2, recommendation A) should be considered in cases of acute abdomen (CQ92)

NSAIDs are as effective as opioids for colic of the biliary tract and may become the first drug of choice (level 1, recommendation B)

NSAIDs should be used in cases of colic due to ureteral stones. When NSAIDs cannot be used, opioids are recommended (level 1, recommendation A)

Table 10 Summary of recommendation: H. Education

CQ107 Are there educational programs that improve practitioner's skill in diagnosing and managing acute abdomen?

A meta-analysis of educational and computer-assisted diagnoses programs aimed at the improvement of practice skill regarding acute abdomen demonstrated improvements in diagnostic precision and decision making (level 3, recommendation $\mathrm{C} 1$ )

CQ108 Is the algorithm for the management of acute abdomen based on current evidence?

Although there is a practice algorithm for the management of childbearing women, the algorithm has been shown to have utility in the exclusion of acute appendicitis, AAA rupture, and extrauterine pregnancy by triage nurses. Further, a computer-aided diagnostic program has been reported; however, the efficacy of the practice algorithm in improving the prognosis of acute abdomen has yet to be validated (level 5 , recommendation $\mathrm{C} 1$ )

\section{(1) Appearance and vital signs}

Patient appearance and the degree of reported pain should be carefully assessed (CQ33). Severe abdominal pain may indicate the risk of mortality. On the other hand, a serious disease may be masked in elderly patients, even in those appearing to have a mild disease.
Regarding posture, patients who refuse to change posture are likely to have peritonitis. Patient contortion may indicate gallstones and ureteral stones (CQ34).

Measured vital signs should include respiratory rate, blood pressure, pulse rate, consciousness level, and body temperature (CQ35). Because the pulse rate may be normal without development of intravascular dehydration during 
Fig. 1 Flowchart for the initial treatment of acute abdomen: a two-step method: Step 1. Differentiate clinical condition and the life-threatening diseases. Confirm patient vital signs, airway $(A)$, breathing $(B)$, circulation $(C)$, and consciousness. Emergency treatment in required if any abnormality is identified during $\mathrm{ABCD}$ assessment. Differentiate super emergent disease and emergent disease while stabilizing the physiologic state of the $\mathrm{ABC}$ of patients with abdominal pain and abnormal $\mathrm{ABCD}$. In patients with abnormal vital signs and in whom emergency tests or radical treatments for the causative disease is challenging, clinicians should not hesitate in requesting a hospital transfer to a specialty institution after securing the airway and initial treatment such as establishing intravenous access routes. Step 2. Evaluation of clinical condition and physical examination. When vital signs are stable, determine whether the cause of the abdominal pain requires surgical intervention according to the findings of a medical history, physical examination, and laboratory findings. In patients with sudden onset or increasing pain, emergency surgery is often required (CQ29)

\section{Step 1 (Check vital signs)}

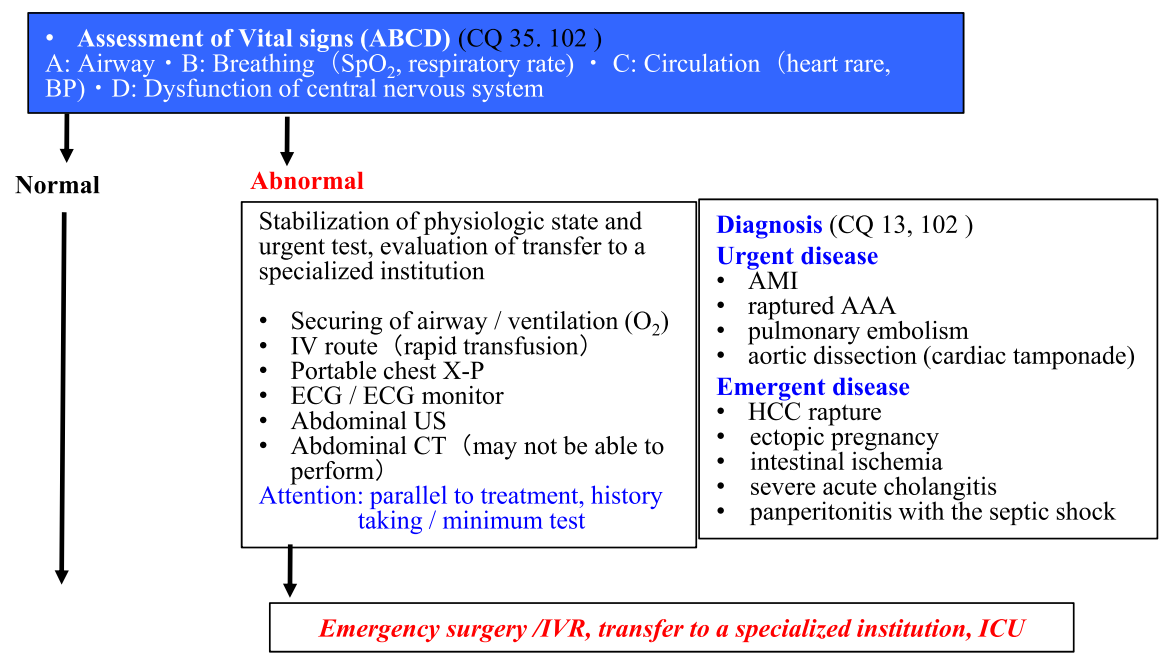

Step 2 (Assessment with history \& physical examination)

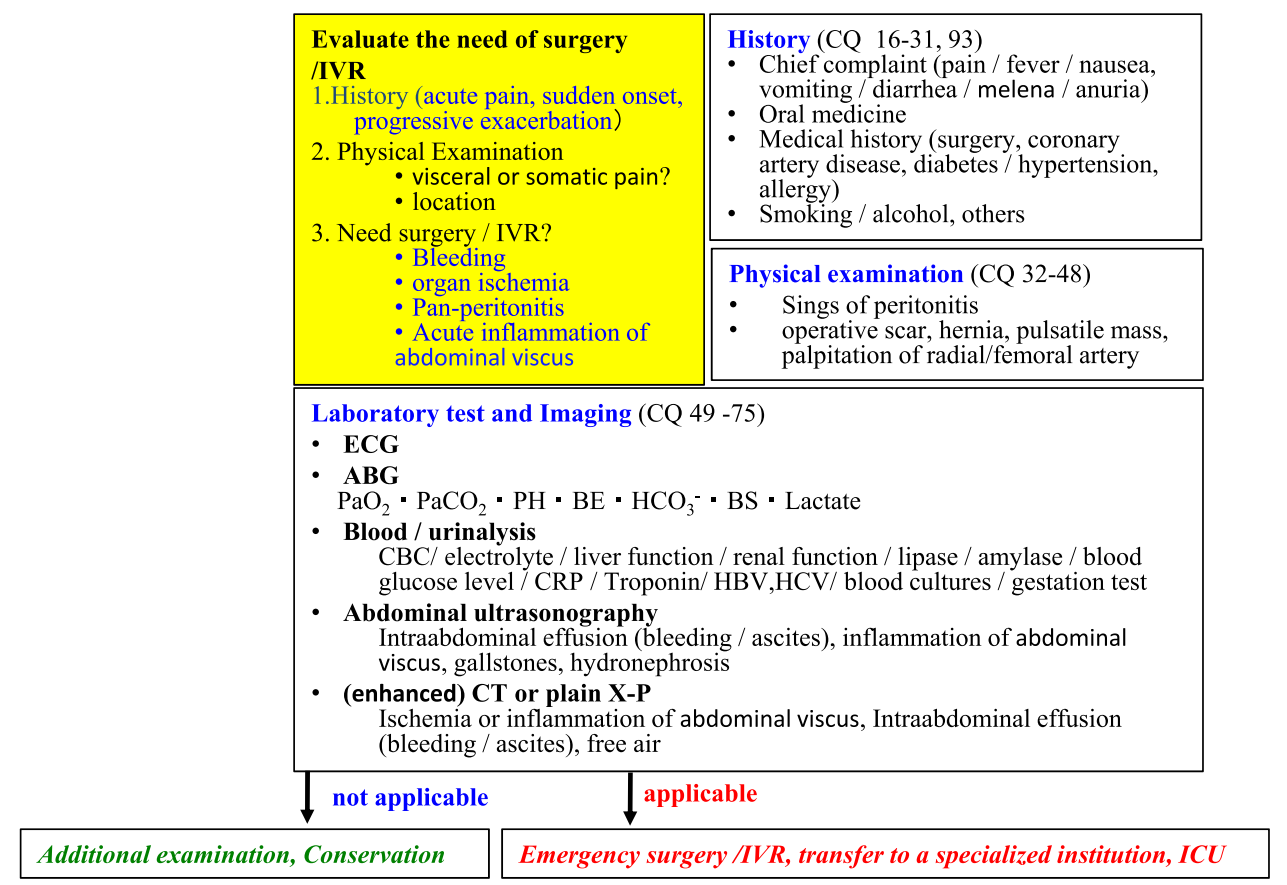

the early stage of bowel obstruction, peritonitis, and intestinal ischemia, the assessment of orthostatic changes in blood pressure and pulse rate has utility in the assessment of patients with acute abdominal pain.

Tachypnea typically indicates intrathoracic disease and metabolic ketoacidosis. Fever may be an important finding; however, serious diseases cannot be excluded in patient with normal temperature, particularly in the elderly, debilitated, or immunocompromised patients.

In addition, anemia, peripheral circulatory failure, and respiratory status should be assessed by physical examination. The conjunctiva should be examined for the presence of jaundice or anemia. Jaundice may also be indicated in the skin. Because pneumonia and myocardial ischemia may be symptomatic in patients with epigastralgia or upper abdominal discomfort, auscultation should be performed to detect respiratory crackles or wheezes, cardiac murmurs, and pleural rubs [42] (level 5).

\section{(2) Medical abdominal examination}

When examining the abdomen with the right hand, it is recommended that the examiner stand on the right side of the patient. It is important to confirm where the 


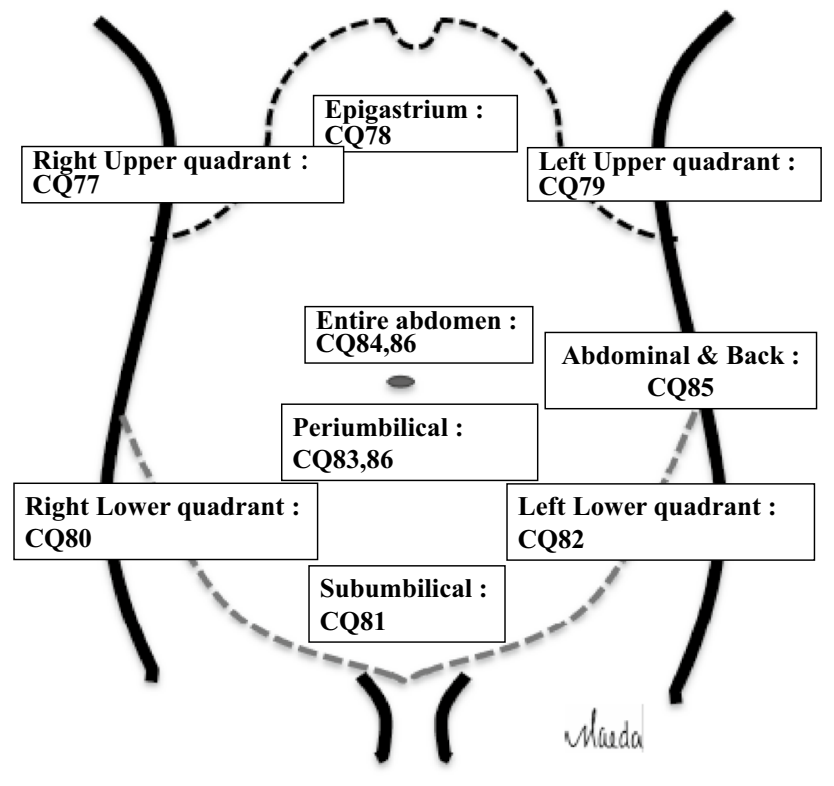

Fig. 2 Differential diagnoses according to the pain location (Q7786)

abdominal pain begins and if there are any changes in pain location. Patients should be adequately exposed from the processus xiphoideus to the groin. Ocular inspection should be performed to detect abdominal surgery scars, herpes zoster infection, stigmata of cirrhosis, hernia, respiratory fluctuations, and skin changes, such as ecchymosis (CQ36).

Percussion and palpation should be gently performed [19] (level 5). Quiet percussion has utility in detecting acute peritonitis (CQ39, 43). If percussion tenderness is positive, it is not necessary for additional examination of rebound tenderness [19] (level 5). Abdominal tension should be removed to identify true muscle guarding (CQ42, 43). Percussion has utility in identifying the presence of ascites and measuring the size of the liver, bladder, and spleen. Tympanic sounds on percussion indicate expanded bowel and dullness indicates the presence of ascites or abdominal masses.

Further medical examination is required at sites of tenderness (CQ76-86, Fig. 2). Muscle guarding is an early peritoneal stimulation sign $(\mathrm{CQ} 42,43)$ and may develop on one side in cases of localized peritonitis. Organ hypertrophy and the presence of masses may be identified by palpation (CQ40). Localized tenderness is usually a reliable evidence of underlying disease in the case of abdominal pain; however, the diagnostic significance of general tenderness is poor. If possible, the size, pulsation, and presence of masses in the liver and spleen should be assessed by palpation and percussion. Pulsatile, distensible mass associated with tenderness is characteristic of abdominal aortic aneurysm (AAA) (CQ40).
Furthermore, patient expression should be observed while evaluating the site and degree of abdominal pain. Patient eye-closing during palpation increases the probability that organic disease is absent (closed eye sign; sensitivity $33 \%$, specificity $93.5 \%$ ) (CQ40) [43] (level 2).

\section{(3) Other practice items}

Assessment of nerve injury, the abdominal wall, and hernial signs is necessary during a comprehensive abdominal examination. Pain in a dermatome distribution with hyperesthesia indicates herpes zoster infection and nerve root injury. Carnett's sign has utility in the differentiation of abdominal wall and intra-abdominal lesions (CQ45). Howship-Romberg's sign may be positive in cases of suspected obturator hernia (CQ41). In men, abdominal pain derived from the testes should also be evaluated.

CQ33 Does a clinician's first impression of a patient have utility in the diagnosis of acute abdomen?

First impression (expression, complexion, respiratory status, conditioning, manner, etc.) provides information regarding the pain site and peritoneal stimulation sign. This information may be used to evaluate the urgency and severity of abdominal pain (level 3, recommendation B).

$<$ Comment $>$ Although general appearance and posture have been reported to have diagnostic value [19] (level 5 ), we were unable to identify any previous study assessing the utility of first impression in the assessment of cases of acute abdomen except for one report of its utility in the detection of severe infection in children [44] (level 3).

\section{CQ35 Does the measurement of vital signs have util- ity in patients with acute abdomen?}

Tachypnea raises the probability of pneumonia, cardiorespiratory failure, and bacteremia (level 2).

Tachycardia, hypotension, and body temperature are correlated with severity and prognosis (level 3) (Table 4).

Therefore, vital signs should always be measured in patients with acute abdomen (recommendation A).

$<$ Comment $>$ Heart rate (rhythm), blood pressure, respiratory rate, temperature, and $\mathrm{SpO}_{2}$ should be included in vital sign assessments. The utilities of individual vital signs in the diagnosis, estimation of severity, and prognosis are presented in Table 11.

\section{(1) Heart rate and blood pressure}

Tachycardia is generally associated with increased morbidity and mortality rates. In patients with septic shock, increased incidence of hospital death is associated with 
Table 11 Utilities of vital signs in acute abdomen

\begin{tabular}{|c|c|c|c|c|}
\hline & Sensitivity $(\%)$ & Specificity $(\%)$ & Positive LR & Negative LR \\
\hline HR $\geq 95 /$ min, predicting mortality in septic shock patients [45] & 97 & 53 & 2.0 & 0.1 \\
\hline $\mathrm{HR} \geq 100 / \mathrm{min}$, predicting complication in gallstone pancreatitis patients [46] & 86 & 87 & 6.8 & NS \\
\hline Systolic BP $\leq 90 \mathrm{mmhg}$, predicting mortality in ICU patients [47] & 21 & 95 & 4.0 & 0.8 \\
\hline Systolic BP $\leq 90 \mathrm{mmhg}$, predicting mortality in septic patients $[48,49]$ & $13-71$ & $85-91$ & 4.9 & NS \\
\hline $\begin{array}{l}\text { Postural pulse increment } \geq 30 \text { /min or severe postural dizziness, predicting mod- } \\
\text { erate acute blood loss }[50]\end{array}$ & $7-57$ & 99 & & \\
\hline $\begin{array}{l}\text { Postural pulse increment } \geq 30 / \text { min or severe postural dizziness, predicting large } \\
\text { acute blood loss [50] }\end{array}$ & 98 & 99 & & \\
\hline $\begin{array}{l}\text { Respiratory rate }>28 / \text { min, detecting pneumonia in patients with cough and fever } \\
\text { [51] }\end{array}$ & $7-36$ & $80-99$ & $2.7(1.4,5.1)$ & $0.9(0.8,0.9)$ \\
\hline Respiratory rate $>20 /$ min with febrile patients, predicting bacteremia [51] & 65 & 30 & NS & NS \\
\hline Acute abdomen with febrile patients, detection of bacteremia [51] & $2-20$ & $90-100$ & 1.7 & NS \\
\hline Fever, detecting peritonitis in the patients with acute abdominal pain [51] & $20-96$ & $11-86$ & $1.4(1.2,1.7)$ & $0.7(0.6,0.8)$ \\
\hline Hyotension, predicting bacteremia in febrile patients [51] & $7-38$ & $82-99$ & $2.2(1.7,2.8)$ & NS \\
\hline Fever, predicting cholecystitis in acute right upper quadrant tenderness [51] & $29-44$ & $37-83$ & NS & NS \\
\hline
\end{tabular}

$N S$ not significant

heart rates $\geq 95 / \mathrm{min}$ [45] (level 4). The incidence of complications is reportedly higher in patients with gallstonepancreatitis with a heart rate $\geq 100 / \mathrm{min}$ [46] (level 3).

Hypotension is associated with poor prognosis [47] (level 3) and strongly associated with the risk of death, particularly in patients with bacteremia [48, 49] (level 3). Postural pulse increase ( $\geq 30 / \mathrm{min}$ ) may indicate acute exsanguination and has greater utility than postural hypotension [50] (level 2).

\section{(2) Respiratory rate}

Respiratory rate is associated with peritonitis, bowel obstruction, and hemoperitoneum. However, respiratory rate should not be more than double the normal rate in patients with abdominal diseases, with higher rates more probable to indicate an intra-thoracic cause [19] (level 5). We were unable to find strong evidence regarding the relationship between respiratory rate and the diagnosis and prognosis of acute abdomen, except for the studies listed in Table 11. Orthopnea may indicate the presence of large quantities of ascitic fluid.

\section{(3) Fever}

The probability of bacteremia is increased in patients with acute abdomen and fever (positive LR, 1.7) [51] (level 2). Furthermore, a study, not limited to patients with acute abdomen, reported that body temperature $<36.5^{\circ} \mathrm{C}$ was associated with increased overall and hospital mortality in patients with bacteremia (positive LR, 2.2) [52] (level 3).
However, when only patients with abdominal pain were evaluated, fever only slightly increased the probability of peritonitis (positive LR, 1.4; negative LR, 0.7) [51] (level 2). Moreover, the presence of fever was unable to discriminate cholecystitis and acute appendicitis from other diseases [51] (level 2), [53] (level 3).

Fever may not be present in elderly patients with cholecystitis and appendicitis, even when complicated by perforation or sepsis [54] (level 5). No additional information is considered to be provided by the measurements of rectal temperature over oral temperature in patients with lower abdominal pain [55] (level 4). Decisions regarding the requirement for surgery in patients with abdominal pain aged $\geq 65$ years should not be based on the presence or absence of fever [56] (level 4). Furthermore, the measurement of body temperature has no utility in the diagnosis or decisions regarding the treatment of acute abdomen.

CQ37 Should auscultation for multiple intestinal peristalsis be performed at a particular location?

Although auscultation is a mandatory component of the abdominal medical examination, there is a lack of consensus regarding the standard method of abdominal auscultation.

If the conduction of peristaltic sounds is good, auscultation at a single location is considered adequate (level 4 , recommendation $\mathrm{C} 1$ ).

Because the clinical significance of auscultation is limited in patients with acute abdomen, in whom peristaltic sounds cannot be heard, it is not recommended that 
multiple auscultations be performed at different locations or for increased durations (level 5, recommendation C2).

$<$ Comment $>$ Auscultation is a mandatory component of the abdominal medical examination and as important as ocular inspection, percussion, or palpation. However, there is a lack of high quality evidence regarding auscultation compared with the other components of abdominal examination. Few clinical studies have evaluated its utility, appropriate duration, site of auscultation, or the interpretation of different abnormal peristaltic murmurs in the medical abdominal examination [57] (level 2).

The majority of peristaltic murmurs are produced by the stomach, with remainder from the large intestine and a small contribution from the small intestine. Because the conduction of peristaltic murmur is very good across the entire abdomen, the source of peristaltic murmur is not always at the site where it is heard. Thorough auscultation across the entire abdomen is not necessary and rarely has clinical value [58] (level 5). A textbook recommends that auscultation be performed at a single location unless an abnormality is detected, in which case auscultation may be performed at several points [57] (level 2).

CQ38 Does abdominal examination using a stethoscope have utility in the diagnosis of acute abdomen?

The identification of abnormal peristaltic murmurs has utility in the diagnosis of bowel obstruction (level 3, recommendation B).

The significance of abdominal bruits is currently unknown in cases of acute abdomen (level 5, recommendation $\mathrm{C} 2$ ).

$<$ Comment $>$ In cases of bowel obstruction, abdominal auscultation has been shown to be clinically useful [51] (level 5). For small intestinal obstruction, the identification of abnormal peristaltic murmur has a sensitivity and specificity of 76 and $88 \%$, respectively, with a positive LR of 6.37 and negative LR of 0.27 . Multivariate analysis demonstrated that abdominal auscultation has the greatest utility in detecting acute abdomen after abdominal distension [59] (level 3).

Judgements regarding the presence of "normal," "decreased," or "asthenic" intestinal murmurs is subjective, and definitive criteria have yet to be established for the evaluation of intestinal murmurs by auscultation. Furthermore, studies have reported a low rate of agreement between observers [60, 61] (level 4), [62-64] (level 3).

The abdominal bruits were reported in $28 \%$ of the patients with AAA (total, 102 patients); however, bruits were also heard in $6.5-31 \%$ of normal adults. In patients with refractory hypertension, the identification of diastolic murmurs in the abdomen during systole has been shown to be useful with positive and negative LRs of 39 and 0.62 , respectively. However, routine auscultation for abdominal bruit has no value in healthy or asymptomatic patients [65] (level 2).

CQ39 Does abdominal percussion have utility in the diagnosis of acute abdomen?

The presence or absence of percussion pain (percussion tenderness) and ascites can be detected by abdominal percussion in cases of acute abdomen (level 2, recommendation A).

$<$ Comment $>$ Abdominal percussion allows the evaluation of percussion pain, ascites, gas, and the presence or absence of hepatosplenomegaly. See CQ42 regarding the use of percussion (percussion tenderness) in detecting peritoneal stimulation.

Tympany over the liver surface may indicate free air due to gastrointestinal perforation in patients with acute abdomen; however, there is a lack of report regarding the sensitivity and specificity of this method [19] (level 5).

Although not performed in patients with acute abdomen, a previous study reported the sensitivity and specificity of flank dullness in detecting ascites as 84 and $54 \%$, respectively. In addition, the reported sensitivity and specificity of shifting dullness in detecting ascites were 77 and $72 \%$, respectively. These findings of ascites may be determined by percussion. However, the use of imaging studies with superior sensitivity and specificity, such as ultrasonography, is recommended for the definite and exclusive diagnosis of ascites [66] (level 2).

CQ40 Does abdominal palpation have utility in the
diagnosis of acute abdomen?

Muscle guarding, muscle rigidity, and rebound tenderness on light palpation are required to confirm the presence of a peritoneal stimulation sign (CQ42, 43) (level 2, recommendation A).

Organ enlargement or abdominal cavity masses such as those in the gallbladder, liver, spleen, or bladder may be detected on deep palpation (level 2, recommendation C1).

$<$ Comment $>$ The width, rather than the strength, of the aortic pulsation can be used to diagnose abdominal aortic aneurysm (AAA). The two determinants of aneurysmal pulsation are (1) aneurysmal size and (2) abdominal circumference. Furthermore, palpation of the abdominal aorta reportedly has no increased risk of AAA rupture during physical examination, as noted in a textbook as follows: "we have not experienced explosion in the medical examination for more than the past 40 years" [67] (level 2).

There have been several studies regarding the sensitivity of palpation for AAA rupture; however, evidence for 
the utility of physical examination in the diagnosis of AAA rupture is unavailable. As the aortic diameter grows larger in cases of AAA rupture compared with that in asymptomatic AAA, it may be assumed that the sensitivity of aortic pulsation increases in such cases. However, studies have shown that the sensitivity of palpation for the detection of AAA rupture varies between 51 and $100 \%$. Because muscle guarding and intestinal expansion due to circulatory disturbance may influence the utility of assessing aortic pulsation, imaging studies should be performed regardless of physical examination findings, when AAA rupture is suspected [67] (level 3).

In a systematic review of acute cholecystitis, the identification of a mass on palpation in the right hypochondrium showed a sensitivity and specificity of 21 and $80 \%$, respectively; however, this was not found to be clinically useful. The sensitivity and specificity of Murphy's sign was reported as 65 and $87 \%$, respectively. But positive and negative LR of the sign for acute cholecystitis is not significant, the imaging modalities such as sonographic Murphy's sign are superior to the sign [68] (level 2).

In a prospective study of the closed eyes sign during abdominal medical examination in 158 patients with undiagnosed acute abdomen, only $6(6.5 \%)$ of the 91 patients with abdominal cavity diseases closed eyes during abdominal examination, whereas $22(33 \%)$ of the 67 patients without abdominal cavity diseases were positive for the closed eyes sign. Therefore, the sensitivity, specificity and positive predictive value (PPV) of the closed eye sign for celiac disease are 93.5, 79 and $79 \%$ respectively. During abdominal palpation, it is necessary to pay attention to the presence or absence of the closed eye sign [43] (level 2).

\section{CQ42 What is the peritoneal stimulation sign?}

The peritoneal stimulation sign occurs when inflammation spreads throughout the abdomen and stimulates the parietal and visceral peritoneum.

Muscle guarding (muscular defense and guarding), rigidity, rebound tenderness, and percussion tenderness should all be evaluated to directly assess the presence of peritoneal stimulation sign.

Indirect evaluations include the cough and heel drop tests.

$<$ Comment $>$ Muscle guarding refers to "voluntary" contraction of the abdominal muscles due to abdominal pain in addition to fear, anxiety, and external low temperature stimuli. The peritoneal stimulation sign refers to "involuntary" contraction of the abdominal muscles in response to peritoneal inflammation that cannot be consciously controlled by the patient. The site of inflammation can be determined by a range of locations where muscle contraction occurs.
To differentiate muscle guarding from peritoneal stimulation sign, pressing the stethoscope into the abdomen during auscultation, pausing, and then repeating again during the medical examination so that the patients do not notice of the test. False muscle guarding decreases as patients relax and may disappear during re-examination [51] (level 5).

Rebound tenderness can be confirmed through patient expression and remarks while pushing with constant force over the peritoneum at the site of pain for several seconds and suddenly releasing the pressure once the patient has become used to the associated pain. Rebound tenderness stimulates the parietal peritoneum and does not necessarily indicate the presence of peritonitis (CQ43).

Pain induced by light percussion is due to the abdominal wall moving in response to percussion [19] (level 5).

The cough and heel drop tests are theoretically the same as the evaluation of percussion pain and can be examined without touching the patient's body. Tests are judged positive if the patient grimaces or moves their hand to their flank during intentional coughing or during heel drop from the standing position.

\section{CQ43 Does peritoneal stimulation have utility in the diagnosis of acute abdomen?}

The peritoneal stimulation sign suggests peritonitis (level 2, recommendation $\mathrm{B}$ ).

If percussion pain is positive, it does not necessarily induce rebound tenderness (level 3, recommendation C2).

Imaging studies are recommended when peritonitis or mesenteric vascular disorder are suspected clinically (level 5 , recommendation $\mathrm{B}$ ).

$<$ Comment $>$ Despite studies demonstrating the utility of the peritoneal stimulation sign in detecting peritonitis, these findings have not been validated by systematic review. Previous studies have predominantly focused on appendicitis; however, there have also been studies evaluating the utility of the peritoneal stimulation sign in detecting ulcer perforation, diverticular perforation, cholecystitis, and pancreatitis [51] (level 3). As the probability of percussion pain is equivalent to rebound tenderness, it is recommended that the evaluation of percussion pain (percussion tenderness and tapping pain) be used rather than rebound tenderness that caused greater pain to patients [51] (level 3).

In the case of adult appendicitis, several systematic reviews and meta-analyses have been reported [69, 70].

Generally, the peritoneal stimulation sign is sensitive for gastrointestinal perforation; however, muscle guarding is reportedly observed in only $21 \%$ of patients aged $\geq 70$ years [71] (level 4 ).

In a prospective study of 124 patients with left-sided diverticulitis, the sensitivity, specificity, positive, and negative LR of pain aggravated by movement was $59 \%, 67 \%$, 
1.81 , and 0.61 , respectively, and those of rebound tenderness were $60 \%, 66 \%, 1.76$, and 0.60, respectively [72] (level 3).

The incidence of abdominal pain and muscle guarding in acute appendicitis has been reported as 95 and $50 \%$, respectively [73] (level 4).

A systematic review of ischemic bowel found that no study has evaluated the specificity and LR of the abdominal pain. The sensitivity of abdominal pain, diffuse tenderness, and the peritoneal stimulation sign for ischemic bowel have been reported as 60-100 \%, 54-90\%, and 13-65 \%, respectively [74] (level 2). The utility of the peritoneal stimulation sign in detecting small intestinal obstruction was not shown [1] (level 3). However, the peritoneal stimulation sign may indicate intestinal ischemia or peritonitis in patients with suspected bowel obstruction [51] (level 5).

CQ45 Does the abdominal wall tenderness test (Carnett's sign) have utility in the diagnosis of acute abdomen?

The abdominal wall tenderness test has utility in diagnosing abdominal wall ache or psychogenic stomachache and excluding intra-abdominal lesions (level 4, recommendation $\mathrm{C} 1$ ).

$<$ Comment $>$ To elicit Carnett's sign, cross both the patient's arms in the dorsal position and ask the patient to hold their head slightly off the bed then apply firm pressure with one had over the spot where pain is strongest while ensuring the abdominal muscles are tense. When tenderness is the same or increased, Carnett's sign is recorded as positive, and when tenderness is decreased, the sign is recorded as negative. Furthermore, abdominal wall tenderness can be assessed by the modified Carnett's sign which is defined as decreased pain on moving from the dorsal position to forward bending while the examiner's hand is placed at the spot of greatest pain $[75,76]$.

The sensitivity, specificity, and positive LR of the abdominal wall tenderness test in detecting peritonitis are reportedly $1-5 \%, 32-72 \%$, and 0.1 , respectively, indicating the utility of this test in the exclusion of intra-abdominal lesions [51] (level 3). When an abdominal wall tenderness test is positive, it is necessary to rule out an abdominal wall muscle hematoma [77] (level 4).

Limitations of the abdominal wall tenderness test include the following [78] (level 5):

1. Patient cooperation may not be obtained in children or elderly patients.

2. The test is considered inadequate in cases of whole abdominal pain.
3. In case of intra-abdominal disease involving the parietal peritoneum, the test becomes positive.

4. There is a risk of rupture in cases of intra-peritoneal abscess.

\subsection{Laboratory tests (Table 6)}

CQ49 Which blood tests have utility in the diagnosis of acute abdomen?

While blood tests have utility in the diagnosis of acute abdomen, history taking, the evaluation of clinical manifestations, physical examination, and imaging studies are also required for fefinite diagnosis. Blood tests that may help in the diagnosis of acute abdomen are shown in Table 12 (level 4).

$<$ Comment $>$ The guidelines of the ACEP (American College Emergency Physicians) in 1994 recommended that clinicians must not rely solely on blood test data for the diagnosis of patients with abdominal pain [79] (level 5). According to the results of emergency visit blood tests of 119,200,000 data of the American NHAMCS (National Hospital Ambulatory Medical Care Survey) reported in 2006, the frequently performed blood tests in patients with abdominal pain are complete blood counts (34.0\%), BUN/ creatinine $(20.1 \%)$, electrolytes $(19.1 \%)$, cardiac enzymes $(19.0 \%)$, liver function tests $(11.5 \%)$, and urinalysis (20.2\%) [80] (level 2).

According to 124 prospective cases reported from a single urban hospital, the proportion of performed blood tests contributing to a diagnosis of acute abdomen was $37 \%$, rising to $41 \%$ after the introduction of a specific policy [81] (level 3).

CQ50 Does the measurement of procalcitonin (PCT)
have utility in the diagnosis and evaluation of acute
abdomen severity?

The utility of PCT in the diagnosis of non-perforated appendicitis has not been shown to be greater than that of CRP or white blood cell (WBC) count; however, PCT has utility in the diagnosis of perforative and abscess formation-related appendicitis. PCT has greater utility in the evaluation of acute abdomen severity of acute pancreatitis and peritonitis (level 2, recommendation B).

$<$ Comment> Previous studies have demonstrated the utility of PCT in the diagnosis of acute abdomen including acute appendicitis, and PCT has been shown to have high precision in the diagnosis of perforation and abscess formation-related appendicitis [82-86] (level 3), [87] (level 4). In a meta-analysis of the sensitivity and specificity of CRP, WBC, and PCT for the diagnosis of acute appendicitis, CRP was found to have the greatest utility in all patients 
Table 12 Blood test for acute abdomen (level 5)

\begin{tabular}{|c|c|c|}
\hline \multirow[t]{7}{*}{ Common blood test usually performed for acute abdomen } & $\mathrm{CBC}$ & WBC, RBC, Hb, Ht, MCV, MCH, MCHC, Plt \\
\hline & Electrolyte & $\mathrm{Na}, \mathrm{K}, \mathrm{Cl}, \mathrm{Ca}$ \\
\hline & Liver & T-Bil, AST (GOT), ALT (GPT), ALP, LDH, (D-Bil) \\
\hline & Kidney & BUN, Cre \\
\hline & Inflammation & CRP \\
\hline & & $\mathrm{CK}$ \\
\hline & Blood sugar & \\
\hline \multirow[t]{9}{*}{ Blood test item for specific disease } & Pancreas & Lipase, amylase \\
\hline & ACS & $\begin{array}{l}\text { (Suspected ACS, epigastric pain), cardiac troponin T, H-FABP, } \\
\text { CPK-MB, (myoglobin) }\end{array}$ \\
\hline & Heart failure & $\mathrm{BNP}$ \\
\hline & Coagulation & $\begin{array}{l}\text { (Suspected coagulation disorder, DIC, Pulmonary embolism or } \\
\text { aortic dissection) PT, APTT, FDP, D-dimer }\end{array}$ \\
\hline & Unconsciousness & $\mathrm{NH}_{3},\left(\right.$ vitamin $\mathrm{B}_{12}$, alcohol $)$ \\
\hline & Infection & $\begin{array}{l}\text { HBs antigen, HBs antibody, an HCV antibody, an examination } \\
\text { for syphilis, an HIV antibody }\end{array}$ \\
\hline & Blood gas analysis & $\begin{array}{l}\mathrm{pH}, \mathrm{PaO}_{2}, \mathrm{PaCO}_{2}, \mathrm{HCO}_{3}^{-}, \mathrm{BE}, \mathrm{Lactate} \text { (evaluation of the over- } \\
\text { all status, intestinal ischemia) }\end{array}$ \\
\hline & Transfusion & Blood type, an irregular antibody \\
\hline & Culture & Blood cultures \\
\hline
\end{tabular}

$A C S$ acute coronay syndrome, $H-F A B P$ heart-type fatty acid-binding protein, $B N P$ Brain natriuretic peptide

Reference [172]

with appendicitis; however, PCT had greater utility in the diagnosis of abscess formation and perforated appendicitis [88] (level 1). Furthermore, PCT has been reported to be correlated with greater degrees of inflammation in cases of appendicitis categorized as normal, phlegmonous, gangrenous, and perforative [83, 87] (level 3).

A systematic review of 24 studies evaluating the severity and presence of infection in cases of pancreatic necrosis reported the sensitivity, specificity, and AUC of PCT in evaluating severity as $80 \%, 91 \%$, and 0.91 , respectively, and for the presence of infection as $72 \%, 86 \%$, and 0.87 , respectively [89] (level 1).

CQ51 In which etiology of acute abdomen does blood gas analysis (including lactate) have utility?

The measurement of base excess (BE), $\mathrm{pH}$, and serum lactic acid level has utility in the diagnosis of shock and intestinal ischemia, and it is commonly performed in patients with high levels of clinical suspicion (level 2, recommendation B).

However, serum lactate levels may not be increased in cases of early intestinal ischemia or volvulus.

$<$ Comment $>$ In a systematic review of 33 studies evaluating serum lactate levels in patients with varying abdominal disease severity, serum lactate levels $>2.5 \mathrm{mM} / \mathrm{L}$ at the time of admission were shown to be associated with poor prognosis [90] (level 1). In a systematic review published in 2011 that analyzed 18 markers from 20 studies and a systematic review of 23 studies published in 2013, lactate, $\mathrm{pH}, \mathrm{BE}, \mathrm{MDCT}$ were shown to have utility in the diagnosis of intestinal ischemia (Table 13) [74] (level 1). However, serum lactate levels rise substantially during the advanced stages of intestinal ischemia and should therefore not be used as an initial diagnostic marker [91]. On the other hand, serum lactate levels may not increase in cases where perfusion is blocked by intestinal twisting, even in cases where ischemia is present (level 2). In such cases, enhanced CT may be required for the evaluation of intestinal ischemia. Enhanced CT findings, such as change in enhancement effect, intestinal wall-thickness, mesenteric congestion, and ascitic quantity have greater utility in the diagnosis of acute abdomen than other markers (CQ68).

Thus, blood gas analyses and particularly serum lactic acid levels represent simple and easy methods with utility in the diagnosis of acute abdomen. The blood gas analyses are also recommended for the assessment of respiratory status of the patient with acute abdomen. In addition, BE and serum lactic acid levels may be measured by gas analysis of venous blood [92] (level 3). However, a greater discrepancy in serum lactic acid levels between arterial blood and venous blood may be observed in patients with severe hepatic dysfunction, and clinicians should be aware of the potential for higher levels in venous samples than in arterial samples [93, 94] (level 3). 
Table 13 Pooled test performance characteristics for laboratory and imaging modalities for the detection of acute mesenteric ischemia

\begin{tabular}{llllll}
\hline & Cut-off value & Sensitivity $(\%)$ & Specificity $(\%)$ & Positive LR & Negative LR \\
\hline Lactate & & $82-90$ & $40-48$ & $2.64-3.04$ & $0.23-0.35$ \\
$\mathrm{pH}$ & $7.35-7.45$ & 38 & 84 & 2.49 & 0.71 \\
$\mathrm{BE}$ & -4.0 to 4.0 & 74 & 42 & 1.26 & 0.62 \\
MDCT & & 94 & 95 & 17.50 & 0.09 \\
\hline
\end{tabular}

References [74, 173]

$L R$ likelihood ratio

Table 14 Urinalysis and disease

\begin{tabular}{|c|c|}
\hline Items & Diseases/clinical conditions to be suspected at positive \\
\hline Protein & $\begin{array}{l}\text { Positive/ } \uparrow: \text { acute glomerulonephritis, chronic nephritis, diabetic nephropathy, nephrotic syndrome, SLE, multiple myeloma, } \\
\text { connective tissue disease, pregnancy-induced hypertension syndrome, dehydration }\end{array}$ \\
\hline Glucose & $\begin{array}{l}\text { Positive/ } \uparrow \text { : diabetes, secondary glycosuria (acute pancreatitis, liver damage, hyperthyroidism, pheochromocytoma, Cushing } \\
\text { syndrome, acromegaly), renal glycosuria, alimentary glycosuria }\end{array}$ \\
\hline $\mathrm{pH}$ & $\begin{array}{l}\text { Acidity: fever, dehydration, starvation, nephritis, diabetes, gout } \\
\text { Alkaline: urinary tract infection, hyperventilation, vomiting, antacid }\end{array}$ \\
\hline Urobilinogen & $\begin{array}{l}\uparrow: \text { Hepatocellular damage, hemolytic anemia, constipation/bowel obstruction, heart failure, fever, etc } \\
\downarrow: \text { Common bile duct obstruction, hepatic jaundice, acute diarrhea, antimicrobial agent, renal failure, etc }\end{array}$ \\
\hline Bilirubin & Hepatocellular damage, intrahepatic cholestasis, extrahepatic cholestasis, etc \\
\hline Ketones & $\begin{array}{l}\text { Severe diabetes, hyperthyroidism, acromegaloidism, Cushing syndrome, long-term fasting state, starvation, hyperfatty diet, } \\
\text { exercise, fever, vomiting, diarrhea, dehydration, etc }\end{array}$ \\
\hline Occult blood & $\begin{array}{l}\text { Microhematuria: renal disease, urinary disease, leukemia, SLE, aortic dissection), } \\
\text { Hemoglobinuria: hemolytic anemia, DIC, incompatible transfusion, paroxysmal nocturnal hemoglobinuria } \\
\text { Myoglobulinuria: rhabdomyolysis, malignant syndrome, excessive exercise, } \\
\text { port urine: porphyria }\end{array}$ \\
\hline Nitrite (bacteria) & Cystitis, pyelitis \\
\hline
\end{tabular}

CQ56 In which patients do urinalysis have utility?

Measurement of urinary human chorionic gonadotropin (hCG) levels has utility in the diagnosis of pregnancy. Measurement of urinary porphobilinogen has utility in the diagnosis of acute porphyria. Urine qualitative analysis has utility in the diagnosis of ureteral stones, urinary tract infection, and ketoacidosis (level 3, recommendation B).

$<$ Comment $>$ Midstream urine is predominantly used for urinalysis; however, obtaining midstream urine is often difficult in patients with an acute abdomen. Urinalysis results may be affected by a number of conditions including exercise, fever, and menstruation. Thus, doctors should ask the patients about their condition at the time of urine sampling to ensure the correct interpretation of results. Generally, protein, glucose, $\mathrm{pH}$, urobilinogen, bilirubin, ketones, occult blood, and nitrites are measured by urinalysis (Table 14; level 5).

Urinary tract infection is common, particularly in women, and bacterial infection is the most frequent in primary care. According to a systematic review, the presence of urinary nitrite has a positive LR of 6.51 and negative LR of 0.58 for the detection of urinary infection in women, whereas the positive LR of symptoms such as frequent urination, pyuria, suprapubic pain, flank pain, and fever ranged between 0.58 and 1.14 [95] (level 1).

However, urinalysis and urine cultures should be performed carefully in elderly patients as bacteriuria is common. According to a clinical review of urinary tract infection in women aged $\geq 65$ years, patients in this population have a wide range of presentations from asymptomatic pyuria to urinary tract infection with symptoms. Asymptomatic pyuria does not require treatment, and treatments for urinary tract infection are typically denied if the urinary leukocyte esterase test is negative. The sensitivity and specificity of urinalysis for urinary tract infection in elderly patients are reportedly 82 and $71 \%$, respectively [96] (level 2).

Hyperglycemia, hyperketonemia, and acidosis are the laboratory findings that characterize ketoacidosis. Ketoacidosis may also be diagnosed, according to the presence of urinary ketones. A prospective study of 516 patients with hyperglycemia among whom 54 had diabetic ketoacidosis, sensitivity, specificity, positive, and negative predictive values (NPV) of urinary ketones were reported as 98.1, 35.1, 15.0, and $99.4 \%$, respectively [97] (level 3). Further, the sensitivity of urinary ketones in a study of 114 patients, 
including those with ketosis and ketoacidosis, was $97 \%$ [98] (level 4). Although the sensitivity of the detection of urinary ketones is high, the measurement of not only urinary ketones but also blood ketones and $\mathrm{HCO}_{3}{ }^{-}$from the venous or arterial blood is required when diabetic ketoacidosis is suspected.

Hematuria, although rare, has been reported in approximately $7 \%$ of patients with aortic dissection at the time when the renal blood flow had been disrupted by stenosis and obstruction of the renal artery [99] (level 4).

\subsection{Imaging tests (Table 7)}

CQ60 In which cases of acute abdomen should plain abdominal radiography be performed?

The diagnosability of plain abdominal radiography is limited and has no value as a part of routine examinations. Even in cases without abnormal findings, ultrasonography (US) or computed tomography (CT) should be considered depending on clinical manifestations (level 2, recommendation C2).

However, in settings without access to US or CT, X-ray is considered in patients suspected to have bowel obstruction, ileus, gastrointestinal perforation, calculus urinary, emphysematous lesion, or a foreign body (level 2, recommendation $\mathrm{C} 1$ ).

$<$ Comment $>$ Because of the simplicity, ease, low involvement, low cost, and the ability to image the whole abdomen, abdominal plain roentgenography has been performed as a routine examination in patients with acute abdomen. However, abnormal findings were found in only 10-20.4\% of patients with acute abdomen in whom abdominal X-ray was performed as a part of a routine examination [100] (level 2), [101-103] (level 3). Only 4-7.2\% of patients found to have abnormal findings, leading to a change in diagnosis or treatment strategy [103, 104] (level 3). Moreover, in cases where abdominal X-rays provided negative or nonspecific findings, other tests revealed abnormal findings in 72 and $78 \%$ of cases, respectively [103] (level 3).

In a study comparing abdominal X-ray and CT in diagnosing the causative disease in cases of acute abdomen, the sensitivity, specificity, and accuracy rate of CT were 96.0, 95.1, and $95.6 \%$ compared with 30.0, 87.8, and $56.0 \%$, respectively, for abdominal X-ray that particularly had a low sensitivity [105] (level 3). In other reports, the sensitivity and specificity of clinical diagnoses for high emergency diseases were 88 and $41 \%$, respectively. In this study, the addition of abdominal X-ray did not increase diagnosability, with sensitivity and specificity remaining at 88 and $43 \%$, respectively. Therefore, US and CT are recommended in such circumstances (CQ65, 66) [106] (level 2).
As intestinal gas patterns, parenteral gas, calcification, and soft-tissue masses are detectable on plain abdominal radiography, plain abdominal radiography has utility in the diagnosis of conditions including bowel obstruction, gastrointestinal perforation, calculus urinary, and foreign bodies [100] (level 2) [102, 105, 107], (level 3). In contrast, plain abdominal radiography has poor utility in the diagnosis of digestive tract bleeding, peptic ulcers, appendicitis, diverticulitis, acute pancreatitis, urinary tract infection, nonspecific abdominal pain, and pain from the pelvic region [99, 105, 107] (level 3). However, a report has demonstrated the utility of plain abdominal radiography when bowel obstruction, gastrointestinal perforation, intestinal ischemia, gall bladder disease, unidentified peritonitis, or calculus urinary are suspected in patients with moderate or severe abdominal pain and tenderness [100] (level 2), [99] (level 3).

\section{CQ62 In which cases of acute abdomen should plain chest radiography be considered?}

When gastrointestinal perforation or diseases of the chest such as pneumonia, pericarditis, or myocardial infarction are suspected, plain chest radiography should be considered (level 3, recommendation B).

$<$ Comment $>$ Plain chest radiography (standing position) is commonly performed at the same time as plain abdominal radiography (standing position or decubitus) as part of an acute abdomen series [101, 102, 104, 105] (level 3). While plain abdominal radiography (standing position) is performed for the evaluation of for gastrointestinal niveau and intra-abdominal free air, plain chest radiography (standing position) is primarily performed to evaluate intraabdominal free air and detect abnormal chest findings.

The diagnosis of intra-abdominal free air on plain abdominal radiography (standing position) is challenging due to the large hepatic nontransmission region. In contrast, free air is easily detected on plain chest radiography (standing position) as the diaphragmatic height is imaged in a near-horizontal state. Plain chest radiography is recommended over abdominal X-ray for the diagnosis of intraabdominal free air [108] (level 4).

Patients with diseases of the chest, such as pneumonia, pericarditis, myocardial infarction, and pulmonary embolism, may present with acute abdomen-like symptoms (CQ76) and were reported to be detected on 10-15\% of plain chest X-rays [101, 104, 109] (level 3).

\section{CQ65 In which cases of acute abdomen is US} required?

US is recommended as a screening test for acute abdomen and strongly recommended particularly when AAA rupture 
or acute cholecystitis is suspected. US is recommended in pregnant women, young woman, or children in whom radioactive exposure is not desirable (level 2, recommendation A). However, expertise in US is required as this technique is highly dependent on practitioner skill (level 2, recommendation $\mathrm{A}$ ).

$<$ Comment> US is simple, easy, can be performed at the bedside, and does not involve radioactive exposure. US has utility as a screening test and for close inspection of the acute abdomen [106, 110-112] (level 2), [113] (level 4), [114] (level 5). US is particularly important in pregnant or young women and children in whom it is desirable to avoid radioactive exposure [114] (level 5). CT cannot be performed in patients with unstable vital signs due to risks associated with patient transfer; however, US can be performed at the bedside.

US has utility in the diagnosis of acute appendicitis, diverticulitis, aortic aneurysm rupture, biliary tract diseases such as cholelithiasis, acute cholecystitis, acute uropathy such as hydronephrosis or renal calculi, and obstetric and gynecological diseases [115-117] (level 3) [19, 118-123] (level 5). In addition, US also has utility in the diagnosis of various disease including gastrointestinal perforation, acute pancreatitis, intraperitoneal abscess, mesenteric artery occlusion, and bowel obstruction [19] (level 4). Further, quick evaluation of ascites and hemoperitoneum, and the evaluation of the intravascular volume of the inferior vena cava, is possible with US [123] (level 5).

For US performed as an initial test for the diagnosis of acute biliary tract disease, the sensitivity, specificity, PPV, and NPV of US have been reported as 83, 95, 75, and $97 \%$, respectively, and shown to be superior to CT that had values of 39, 93, 50, and $89 \%$, respectively, when used for initial testing [115] (level 3). In the diagnosis of acute appendicitis, the sensitivity, specificity, and accuracy rate of US was reported as 75-90\%, 95-100\%, and 90-95\%, respectively, whereas CT had values of 90-100\%, 91-99\%, and 94-98\%, respectively. US is recommended in children, pregnant patients, and premenopausal women with frequent gynecological presentations resembling appendicitis [116] (level 3).

In a diagnostic accuracy study of urgent abdominal cases, false positives, according to clinical diagnosis, and $\mathrm{X}$-ray were significantly reduced by performing US or enhanced CT [106] (level 2). However, the sensitivity of addition of US alone was $70 \%$, whereas that of CT alone was $89 \%$, indicating the limitation of US for the diagnosis of emergent presentations. However, the initial use of US alone is recommended in cases where diseases that only US can detect are suspected or when radiation exposure is undesirable. CT is recommended when no obvious abnormality can be found or when a definite diagnosis cannot be made.

In a RCT evaluating the use of US by surgeons in the emergency room who had received constant training, the correct diagnosis rate with US was significantly higher than that with physical examination and laboratory testing, and US was shown to significantly reduce hospitalization and the need for specialist US [111, 112] (level 2). Further, US has utility in informing decisions regarding the need for surgical interventions, indicting the importance of US education for both surgeons and ER doctors.

CQ66 In which cases of acute abdomen should CT be
performed?

CT may be indicated in all patients with acute abdomen (level 2, recommendation A). However, CT can be omitted when a diagnosis is made according to the results of precedent examinations such as US. Radiation exposure should be considered with the use of CT.

$<$ Comment $>$ Due to the short testing time, lack of reliance on operator skill, and the wide use of CT (and MDCT, in particular), there have been many studies evaluating the utility of CT in the diagnosis of acute abdomen [106, 124, 125] (level 2) [117, 126, 127], (level 3). In contrast, the use of CT is limited by associated radiation exposure [128] (level 4).

The sensitivity of CT in the diagnosis of acute abdomen was found to be $90 \%$, significantly higher than that of clinical diagnosis (76\%), and treatment strategies were changed according to the results of CT in 25 of 91 patients with acute abdomen [126] (level 3). Treatment strategies were changed in 33 of 55 patients in a separate study of patients with acute abdomen. Further the hospitalization rate was decreased by $23.8 \%$, and quick surgery was made possible by the use of CT [124] (level 2).

The results of abdominal physical examination and laboratory testing often fail to reflect the underlying causes of acute abdomen in elderly patients. The accuracy rate of $\mathrm{CT}$ in the diagnosis of acute abdomen in elderly aged $\geq 75$ years was found to not significantly differ with or without clinical information and laboratory data. Therefore, a number of studies have posited the use of CT to diagnose acute abdomen without waiting for laboratory data to allow more earlier treatment [127] (level 3).

The sensitivity, specificity, and accuracy rate of nonenhanced CT in diagnosing acute abdomen is reportedly 96.0, 95.1, and $95.6 \%$, respectively, significantly better than with plain X-ray $(30.0,87.8$, and $56.0 \%$, respectively) [125] (level 2). When comparing the utility of US 
and enhanced CT in diagnosing appendicitis and diverticulitis, the sensitivity of enhanced CT was found to be 94 and $81 \%$, respectively, significantly higher than US (76 and $61 \%$, respectively) [117] (level 3).

CQ70 In patients with abdominal pain and no abnormality detected on $\mathrm{CT}$, which emergent diseases can be excluded?

CT has high diagnosability for intestinal ischemia, gastrointestinal perforation, acute appendicitis, diverticulitis, biliary tract calculus, and acute pancreatitis among others. However, these diseases cannot be completely ruled out, even if abnormalities are not found on CT (level 3, recommendation B).

$<$ Comment $>$ Enhanced CT reportedly has a sensitivity and specificity of 93.3 and $95.9 \%$ indicating a high diagnosability for intestinal ischemia by imaging in the arterial and venous phase (2 phases) (CQ68). The diagnosability of $\mathrm{CT}$ for gastrointestinal perforation is also high. CT is able to detect the presence of free air in the upper gastrointestinal tract perforation in more than $85 \%$ of cases, and the perforation site can reportedly be identified in $80.5 \%$ of cases if a rearrangement image of 1-mm thickness degree was used (CQ71, 72).

It is reported that the sensitivity and NPVs of CT in diagnosing acute appendicitis and ureteral stones $\mathrm{CT}$ are all $90 \%$ or greater $[129,130]$ (level 3). A high diagnosability of CT has also been reported for cholelithiasis with a sensitivity and NPV of 73 and $96 \%$, diverticulitis (81 and $98 \%$, respectively) [117] (level 3), choledocholithiasis (97 and $94 \%$, respectively) [131] (level 3), and acute pancreatitis (77.8 and $81.8 \%$, respectively) [132] (level 3).

The majority of diseases mentioned here can be ruled out with relative confidence in cases where no abnormal findings are detected on CT. However, as these diseases cannot be completely excluded, clinical information aside from the results of imaging should be considered to arrive at a final diagnosis.

\section{CQ72 What is the role of abdominal US and CT in} cases of peritonitis due to acute abdomen?

The diagnosis of peritonitis due to acute abdomen is possible from medical history, physical examination, and laboratory testing in the majority of cases; however, imaging studies should be performed to confirm peritonitis and the underlying cause of investigation.

US is the initial imaging study of choice; however, enhanced abdominal or pelvis CT (when possible, plain+enhanced) should be performed in cases where a definitive diagnosis has not been reached with US (level 4, recommendation $\mathrm{B}$ ).
$<$ Comment $>$ Peritonitis may result from infectious and noninfectious causes and may accompanied by bleeding in a proportion of cases. As ascites are exudative, the echo brightness of peritonitis is higher than leaking ascites, and CT density tends to be high and often accompanied by strings and debris. In cases of bleeding, CT findings are typically more remarkable. The peritoneum reflects the presence of inflammation, thickens, and displays an enhance effect after contrast media administration. Inflammation may be localized or widely disseminated and is often complicated with localized or extensive ileus.

In cases of idiopathic peritonitis (predominantly due to the infection of pre-existent ascites), the route of infection is unclear, and secondary peritonitis may develop as a complication of another primary disease (acute appendicitis, $37 \%$; acute cholecystitis, $13.4 \%$; postoperative, $15.9 \%$; diverticulitis, $7.7 \%$; large bowel perforation, $7.3 \%$; gastroduodenal perforation, $7.3 \%$; and intestinal perforation, $4.8 \%$ ) [133] (level 2). The differentiation of idiopathic and secondary peritonitis and the identification of the original disease has utility in informing clinical decisions regarding treatments for peritonitis and can often be provided by imaging studies.

The appropriateness of imaging test varies according to the suspected disease of origin. US has the greatest utility for assessment of the gallbladder for the presence of bile duct disease. For acute appendicitis, enhanced CT has been shown to have high reliability and, particularly, high diagnosability in cases of perforated appendicitis [134] (level 3).

In contrast, US has greater utility in children and thin adults. Enhanced CT is recommended for cases of suspected acute pancreatitis and alimentary tract diseases such as diverticulitis, gastrointestinal perforation, intestinal obstruction, and intestinal ischemia. Magnetic resonance imaging has utility when used in conjunction with $\mathrm{CT}$ in detecting gynecological diseases such as pelvic inflammation, extrauterine pregnancy, ovarian torsion, and ovarian hemorrhage.

CQ74 How great is the risk of radiation exposure for pregnant woman and children?

The incidence of fetal malformation and central nerve disorders does not increase with radiation exposure (less than $50-100 \mathrm{mGy}$ ) in pregnant women (level 3).

There is a greater risk of carcinogenesis to fetuses and children than that to adults (level 3) (Table 9).

$<$ Comment> "The Guidelines Obstetrical Practice in Japan 2014," state radiation exposure less than 50 mGy does not increase the incidence of fetal malformation during organogenesis (day 11-10 weeks) (Table 15). Further, $100 \mathrm{mGy}$ has no effect on pregnancies after 10 weeks gestation [135] (level 5). The International Commission on 
Table 15 A fetal radiation dose of each radiation image

\begin{tabular}{llll}
\hline Radiation image & & Mean radiation dose (mGy) & Max radiation dose (mGy) \\
\hline Plain X-P & Head & $\leq 0.01$ & $\leq 0.01$ \\
& Chest & $\leq 0.01$ & $\leq 0.01$ \\
& Abdomen & 1.4 & 4.2 \\
& Lumbar vertebrae & 1.7 & 10 \\
& Pelvis & 1.1 & 4 \\
& Intravenous urography & 1.7 & 10 \\
Gastrointestinal series & Upper gastrointestinal tract & 1.1 & 5.8 \\
& Lower digestive tract & 6.8 & 24 \\
CT & Head & $\leq 0.005$ & $\leq 0.005$ \\
& Chest & 0.06 & 0.96 \\
& Abdomen & 8.0 & 49 \\
\end{tabular}

Reference [135]

Radiological Protection 84 stated fetal radiation exposure of less than $100 \mathrm{mGy}$ at any time during cannot be a reason for pregnancy termination [136] (level 5).

In contrast, there has been a report of increased incidence of malformations in between 4 and 10 weeks gestation (organogenesis) with radiation exposure of $100 \mathrm{mGy}$ or greater [137] (level 5). Further, a separate study found severe mental retardation occurred with radiation exposure of $500 \mathrm{mGy}$ or more [138] (level 3). However, the average fetal radiation dose of abdominal CT is reportedly $8 \mathrm{mGy}$, with a maximum of $49 \mathrm{mGy}$ [136] (level 3); meaning exposures of greater than $100 \mathrm{mGy}$ can occur without multiple exposures. Therefore, the radiation exposure associated with CT may be acceptable after having considered equivalent tests, with the benefit outweighing the risks even in pregnancy.

The risk of the carcinogenesis due to radiation exposure of $100 \mathrm{mSv}$ or less is thought to be extremely low in adults; however, fetuses and children are susceptible to radiation, and there have been reports of the risk of carcinogenesis $[136,139,140]$ (level 3). It is estimated that fetal radiation exposure of $10 \mathrm{mGy}$ may be associated with a $40 \%$ increased incidence of childhood cancer; however, this raises the overall incidence from 0.2 to $0.3 \%$ to 0.3 to $0.4 \%$, equivalent to one extra cancer death per 1700 [136] (level 5).

\subsection{Differential diagnosis (Table 8)}

CQ76 What diseases other than abdominal or retroperitoneal may be confused with acute abdomen?

Differential diagnoses are listed in (Tables 16, 17, 18) (level 5).

$<$ Comment $>$ Many reviews have described similarities between acute abdomen and other diseases and provided classifications according to mechanism of disease or anatomical locations [19, 141, 142] (level 5).

Diseases classified according to anatomy and underlying mechanism are shown in Table 17 [142] (level 5).

CQ89 What are the characteristic, prognostic, and predictive features of acute abdomen in elderly patients?

Regarding the characteristics of acute abdomen in elderly patients, the results of abdominal physical examination and laboratory testing often do not reflect the patient condition, decisions regarding surgical indications and prognosis are challenging (level 3), and a definitive diagnosis is often not possible (level 3). As CT has utility in the diagnosis of acute abdomen and informing decisions regarding treatment strategies, abdominal $\mathrm{CT}$ is recommended as required (level 3, recommendation B).

Urgent hospitalization and surgical intervention are often required for elderly people complaining of abdominal pain (level 3).

Further, mortality rates increase with patient age.

Acute intestinal ischemia, AAA rupture, and organ dysfunction of the extra-abdominal cavity are all associated with high mortality rates (level 3).

$<$ Comment $>$ Laurell et al. divided patients with emergency visits for abdominal pain into three groups according to age: 20-64 years old (control group, $n=1,458$ ); 65-79 years old (elderly people group; $n=557$ cases); and $\geq 80$ years old (super elderly people group, $n=274$ cases) [143] (level 3). The probability of misdiagnosis in the emergency room in patients aged $\geq 65$ years was significantly higher at $52 \%$ compared with the control group at $45 \%$. The authors posited that physical examinations were 
Table 16 Differential diseases for acute abdomen other than abdominal and retroperitoneal disease

\begin{tabular}{|c|c|c|}
\hline \multirow[t]{5}{*}{ Extra-abdominal cavity } & Cardiovascular & $\begin{array}{l}\text { Acute coronary syndrome, endocarditis, pericarditis, myocarditis, } \\
\text { aortic dissection, aortic aneurysm rupture }\end{array}$ \\
\hline & Respiratory & $\begin{array}{l}\text { Pneumonia, pleuritis, empyema, pneumothorax, pulmonary throm- } \\
\text { boembolism }\end{array}$ \\
\hline & Esophageal & Esophageal rupture, esophagus spasm, esophagitis \\
\hline & Musculoskeletal & $\begin{array}{l}\text { Radiculopathy, spinal cord/peripheral nerve tumor, spinal osteoar- } \\
\text { thritis, herniated disk, Diskitis, iliopsoas muscle abscess, osteo- } \\
\text { myelitis, slipping rib syndrome, costochondritis, mondor disease, } \\
\text { acnes (abdominal cutaneous nerveentrapment syndrome) }\end{array}$ \\
\hline & Groin, pubic region & $\begin{array}{l}\text { Torsion of spermatic cord, epididymitis, (inguinal, femoral, obtura- } \\
\text { tor) hernial (incarceration), haemorrhoids, anal fistula }\end{array}$ \\
\hline \multirow[t]{4}{*}{ Systemic disease } & Hematological, allergic, connective tissue disease & $\begin{array}{l}\text { Acute leukemia, hemolytic anemia, sickle cell disease, lymphoma, } \\
\text { systemic lupus erythematosus, rheumatoid arthritis, dermato- } \\
\text { myositis, polyarteritis nodosa, iga vasculitis (Henoch-Schonlein } \\
\text { purpura), food allergy, angioedema, eosiophilic enteritis }\end{array}$ \\
\hline & Endocrine, metabolic & $\begin{array}{l}\text { Acute adrenal insufficiency, diabetic ketoacidosis, hyperthyroidism, } \\
\text { porphyria, uremia }\end{array}$ \\
\hline & Poisoning & Drug allergy (insect/spider stab, snake toxin), lead poisoning \\
\hline & Infection & $\begin{array}{l}\text { Streptococcal sore throat, herpes zoster, varicella, osteomyelitis, } \\
\text { typhoid fever, tuberculosis, brucellosis, toxic shock syndrome }\end{array}$ \\
\hline Others & \multicolumn{2}{|c|}{$\begin{array}{l}\text { Acute glaucoma, abdominal epilepsy, abdominal migraine, mental disorder, foreign body, heat stroke, familial Medi- } \\
\text { terranean fever, gynecological (ovulation pain) }\end{array}$} \\
\hline
\end{tabular}

Table 17 Mechanisms of abdominal symptoms in extra-abdominal and systemic diseases (some diseases cause abdominal symptoms by several mechanisms)

\begin{tabular}{lc}
\hline Mechanism & Examples \\
\hline $\begin{array}{l}\text { Systemic disease causes pathologic condition in intra-abdominal } \\
\text { organs }\end{array}$ & $\begin{array}{c}\text { Heavy metal toxicity, tuberculosis, CHF, spider envenomation, } \\
\text { alcoholic ketoacidosis, sickle cell infarction and biliary disease, } \\
\text { neutropenia, hypercalcemia (pancreatitis, ileus, gastritis), CI inhibitor } \\
\text { deficiency, SLE (lupus enteritis) }\end{array}$ \\
$\begin{array}{l}\text { Systemic disease is precipitated by pathologic condition in intra- } \\
\text { abdominal organs }\end{array}$ & $\begin{array}{l}\text { Hypercalcemia and hypocalcemia, sickle cell painful crisis. SLE } \\
\text { Systemic disease causes nausea, vomiting, or other gastrointestinal } \\
\text { symptoms }\end{array}$ \\
$\begin{array}{l}\text { Extra-abdominal disease causes abdominal symptoms by neural } \\
\text { mechanisms or with functional or poorly understood organic basis }\end{array}$ & $\begin{array}{c}\text { DKA, glaucoma, thyrotoxicosis, porphyria, hypercalcemia (neuropa- } \\
\text { thy, hypo motility), hypocalcemia, adrenal crisis, gonadal torsion, } \\
\text { pheochromocytoma }\end{array}$ \\
$\begin{array}{c}\text { Disease of extra-abdominal organs causes perception of pain in the } \\
\text { abdomen because of irritation of contiguous extra-abdominal struc- } \\
\text { tures }\end{array}$ & $\begin{array}{c}\text { Lower lobe pneumonia, pulmonary emboli, pleuritis, inferior wall } \\
\text { cardiac ischemia, pyelonephritis, spinal and other musculoskeletal } \\
\text { diseases, testicular torsion }\end{array}$ \\
$\begin{array}{l}\text { Abdominal pain due to referred pain from extra-abdominal structures } \\
\text { ACS and other diseases of mediastinal structures, ureterolithiasis, } \\
\text { pyelonephritis }\end{array}$ \\
\hline
\end{tabular}

$A C S$ acute coronary syndrome, $C H F$ congestive heart failure, $D K A$ diabetic ketoacidosis, SLE systemic lupus erethematosus

Reference [142]

not clear in elderly patients, and blood tests were less likely to demonstrate abnormalities.

Regarding physical examination at diagnosis, rebound tenderness (control group, $62 \%$; elderly people, $35 \%$; super elderly people, $29 \%$ ) was found to be associated with peritonitis in patients aged $\geq 65$ years $(P<0.001)$. Lower rates of abdominal muscle guarding $(62,35$, and
$29 \%$, respectively, $P=0.003)$ and rectal tenderness (32, 17 , and $18 \%$, respectively, $P=0.004$ ) were observed in patients aged $\geq 65$ years compared with the control group.

When comparing CRP levels between patients requiring surgery and patients not requiring surgery in patients aged $\geq 65$ years, no differences in CRP at admission were observed between the two groups and compared with 
younger generations. As a result, hospital mortality was worse in elderly, with rates of 4.7 and $1.8 \%$ in the super elderly and elderly group, respectively, compared with $0.1 \%$ in the control group. Acute intestinal ischemia, AAA rapture, organ dysfunction of the extra-abdominal cavity, pancreatitis, cholelithiasis, and large bowel obstruction are all more likely to be causes of death in elderly patients compared with young people.

The utility of abdominal CT in diagnosing abdominal disease has been reported by large number of studies.

A prospective cohort study of elderly people with abdominal pain demonstrated diagnosis and treatment strategies can change a result of abdominal CT [144] (level 3). Abdominal CT was performed in $37 \%$ of elderly patients with abdominal pain and had utility in informing a diagnosis in $57 \%$ of these patients [145] (level 3).

Factors requiring surgery in elderly patients aged $\geq 65$ years with abdominal pain are reportedly hypotension, abnormal peristaltic sounds, significant abnormal findings on radiographic examination, bowel loop expansion on abdominal X-ray, and leukocytosis. Factors associated with increased risk of death were found to be the presence of "free air in the abdominal X-ray," "age > 84 years," "significant abnormal findings in the radiographic examination," and "neutrophilia" [146] (level 3).

Considering the above-mentioned characteristic of elderly patients with abdominal pain and potential for disease to easily become serious, Lion and Clark compiled a list of differential diagnoses (Table 19) and points to keep in mind (Table 20) at diagnosis based on many references [147] (level 5).

\subsection{Initial therapy (Table 9)}

CQ102 What is initial treatment when acute abdomen is suspected?

First, the patient's vital signs and airway (A), breathing (B), circulation $(\mathrm{C})$, and consciousness, should be confirmed, and emergency treatment provided upon the determination of abnormal vital signs and $\mathrm{ABC}$ and treatment for the causative disease, if known, should be initiated immediately. When radical treatment is challenging, emergency treatment should be provided and hospital transfer should be considered (Fig. 1) (level 4, recommendation A).

When vital signs are stable, decisions regarding the need for emergency surgery should be made based on medical history and abdominal findings. In addition, laboratory tests and imaging studies have utility in determining whether the clinical condition (bleeding, organ ischemia, pan-peritonitis, acute inflammation) requires surgical intervention (Fig. 1) (level 4, recommendation A).
CQ103 How should initial transfusions be initiated in cases of acute abdomen?

Initial transfusions should be initiated immediately when intraperitoneal infections are diagnosed, even if circulatory dynamics are stable (level 3, recommendation A).

The stabilization circulatory dynamics has top priority in cases of shock (level 5, recommendation A).

Crystalloid solutions, such as Ringer's solutions, should be used (level 1, recommendation A).

Hydroxyethyl starch (HES) is not recommended (level 1 , recommendation $\mathrm{D})$.

The use of albumin preparation should be considered in patients in shock and requiring large transfusion volumes or who have hypoalbuminemia (level 1 , recommendation C1).

Erythrocyte transfusion should be provided with hemoglobin levels less than 7-9 g/dl (level 1, recommendation A).

$<$ Comment $>$ Patients with acute abdomen commonly present with dehydration and appetite loss, fluid intake reduction due to nausea, increased water excretion due to vomiting and diarrhea, and increased insensible perspiration due to the fever. Historically, an advantage of transfusions was reported in cases perforative or abscess formation-related appendicitis, even if not complicated with septic shock [148] (level 3). When intra-abdominal infection is suspected, even if circulatory dynamics are stable, immediate transfusion is recommend by the intraabdominal infection guidelines (SIS and IDSA guidelines) [149] (level 3). In cases of hemorrhagic shock or septic shock caused by intra-abdominal infection, the stabilization of circulatory dynamics with rapid transfusion (blood transfusion as required) is the top priority [150, 151] (level 5).

Albumin preparations were not found to significantly reduce mortality or adverse event rates compared with crystalloid solution [152, 153] (level 1); however, the cost of albumin preparations is substantially higher. Therefore, crystalloid solution should be used initially; however, albumin preparations may be considered in cases requiring large transfusion volumes or with hypoalbuminemia [150, 151] (level 5).

In a clinical trial of HES, the efficacy of maintaining infusion volumes of HES to stabilize circulatory dynamics was found to be greater than with Ringer's solution and saline; however, it was reported that the rate of adverse events, such as renal failure and bleeding, significantly increased without improvements in mortality [154, 155] (level 1). Accordingly, HES should not be used to treat hypovolemia or septic shock due to acute abdomen. 
Table 18 Differential diagnosis for abdominal pain

CQ77 What is the differential diagnosis for RUQ pain?

Common: esophagus, stomach/duodenal disease, hepato-biliary disease is common (Level 3)

Gastrointestinal (GI)

Cholecystitis, cholelithiasis, cholangitis, colitis, diverticulitis, appendicitis, liver abscess, hepatitis, liver masses, gastric/duodenal ulcer, pancreatitis

Vascular

Acute coronary syndrome, myocarditis, endocarditis, pericarditis, aortic dissection, superior mesenteric artery occlusion/dissection

Urinary

Nephrolithiasis, pyelonephritis, ureteral stone, renal infarction, nephrolithiasis, and others

Respiratory

Pneumonia, pulmonary embolism, empyema

Others

Fitz-Hugh-Curtis syndrome

(Level 5)

CQ78 What is the differential diagnosis for epigastric pain?

Common: esophagus, stomach/duodenal disease, hepato-biliary disease is common (Level 3)

GI

Vascular

Urinary

Others

Gastric ulcer, duodenal ulcer, bowel obstruction, colitis, diverticulitis, appendicitis, cholecystitis, cholelithiasis, cholangitis, liver abscess, hepatitis, liver masses, pancreatitis

Acute coronary syndrome, myocarditis, endocarditis, pericarditis, aortic dissection, superior mesenteric artery occlusion/dissection

Nephrolithiasis, pyelonephritis, ureteral stone, renal infarction, adrenal infarction

Respiratory disease (pneumonia, pulmonary embolism, empyema)

(Level 5)

CQ79 What is the differential diagnosis for LUQ pain?

GI

Esophageal rupture, esophagitis, esophageal spasm, gastric ulcer, gastritis, splenic infarction, splenomegaly, splenic rupture, splenic abscess, spleen twisting, splenic aneurysm, diverticulitis, ischemic enteritis, bowel obstruction, left-sided appendicitis, pancreatitis, pancreatic tumor

Vascular

Acute coronary syndrome, myocarditis, endocarditis, pericarditis, aortic dissection, superior mesenteric artery dissection/occlusion

Left kidney/adrenal gland

Renal infarction, adrenal infarction, pyelonephritis, nephrolithiasis, ureteral stone

Others

The disease in the left chest (left lower lung pneumonia, left pneumothorax, left empyema)

(Level 5)

CQ80 What is the differential diagnosis for RLQ pain?

Common: intestinal disease, urinary disease, gynecological disease (Level 3)

GI

Appendicitis, colitis, diverticulitis, inflammatory bowel disease, irritable bowel syndrome, cholecystitis, pancreatitis, inguinal hernia

Urinary

Prostatitis, epididymitis, ureteral stone, urinary tract infection

Obstetrics and gynecological disease Extrauterine pregnancy, endometriosis, ovarian hemorrhage, ovarian cyst raputure, torsion of ovary, uterine myoma, pelvic peritonitis, appendicular abscess (fallopian tubes/ovarian abscess), adnexitis

Vascular

Aortic dissection, aneurysm rupture

Others

Iliopsoas abscess, retroperitoneal haemorrhage

(Level 5)

CQ81 What is the differential diagnosis for subumbilical pain?

Common: intestinal disease, urinary disease, gynecological disease (Level 3)

GI

Urinary

Obstetrics and gynecological

Appendicitis, colitis, diverticulitis, inflammatory bowel disease, irritable bowel syndrome

Cystitis, ureteral stone, pyelonephritis, urinary retention

Extrauterine pregnancy, endometriosis, uterine myoma, ovarian tumor, torsion of ovary, pelvic peritonitis, ovarian hemorrhage

(Level 5)

CQ82 What is the differential diagnosis for LLQ pain?

Common: intestinal disease, urinary disease, gynecological disease (Level 3)

GI

Urinary

Obstetrics and gynecological
Onstipation (obstruction by the stool), bowel obstruction (including hernia incarceration), bowel tumor, colitis (infective, ischemic), inflammatory bowel disease, bowel infection, diverticulitis

Prostatitis, epididymitis, ureteral stone, urinary tract infection

Extrauterine pregnancy, endometriosis, ovarian hemorrhage, ovarian cyst rapture, torsion of ovary, uterine myoma, pelvic peritonitis, appendicular abscess (fallopian tubes/ovarian abscess), adnexitis 
Table 18 continued

\begin{tabular}{|c|c|}
\hline Vascular & Aortic dissection, aneurysm ruptured \\
\hline Others & $\begin{array}{l}\text { Iliopsoas abscess, retroperitoneal haemorrhage } \\
\text { (Level 5) }\end{array}$ \\
\hline \multicolumn{2}{|c|}{ CQ83 What is the differential diagnosis for periumbilical pain? } \\
\hline \multicolumn{2}{|c|}{ Common: esophagus, stomach/duodenal disease, hepato-biliary system (Level 3) } \\
\hline GI & Acute appendicitis (initial symptoms), small intestinal obstruction, simple bowel colic, pancreatitis \\
\hline Vascular & $\begin{array}{l}\text { Mesenteric artery occlusion, acute coronary syndrome, abdominal aortic aneurysm, splanchnic artery } \\
\text { dissection }\end{array}$ \\
\hline Others & $\begin{array}{l}\text { Tabes dorsalis, acute glaucoma, urachal remnant } \\
\text { (Level 5) }\end{array}$ \\
\hline \multicolumn{2}{|c|}{ CQ84 What is the differential diagnosis for generalized abdominal pain? } \\
\hline Vascular & Aortic aneurysm rupture, aortic dissection, mesenteric artery occlusion, mesenteric vein thrombosis \\
\hline GI & $\begin{array}{l}\text { Gastrointestinal perforation/obstruction (strangulated), acute gastritis, acute enteritis, rupture of abdomi- } \\
\text { nal organ, pancreatitis }\end{array}$ \\
\hline Endocrine, metabolic & Diabetic ketoacidosis, alcoholic ketoacidosis, acute porphyria \\
\hline Others & $\begin{array}{l}\text { Poisoning (lead, arsenic, ets), iga vasculitis (Henoch-Schonlein purpura), bilateral pneumonia } \\
\text { (Level 5) }\end{array}$ \\
\hline \multicolumn{2}{|c|}{ CQ85 What is the differential diagnosis for abdominal and back pain? } \\
\hline \multicolumn{2}{|c|}{ Should be careful about retroperitoneal lesions } \\
\hline Vascular & Aortic aneurysm rupture, aortic dissection \\
\hline GI & Acute pancreatitis (chronic pancreatitis), cholelithiasis, acute cholecystitis, splenic infarction \\
\hline Urinary & Renal/ureteral stone, renal infarction \\
\hline Others & $\begin{array}{l}\text { Herpes zoster, compression fracture, iliopsoas abscess } \\
\text { (Level 5) }\end{array}$ \\
\hline
\end{tabular}

CQ86 What is the differential diagnosis for severe central abdominal pain with shock?

Acute pancreatitis, superior mesenteric artery occlusion, haemoperitoneum, aortic aneurysm rupture, aortic dissection, gastrointestinal perforation, intestinal necrosis, acute coronary syndrome, extrauterine pregnancy (women)

(Level 5)

In a multicenter RCT evaluating transfusion criteria for severe disease, no significant difference in mortality was observed between the liberal transfusion group (transfusion initiated at less than $\mathrm{Hb} 10 \mathrm{~g} / \mathrm{dl}$ to a target of $10-12 \mathrm{~g} / \mathrm{dl}$ ) and restrictive transfusion group (transfusion initiated at less than $\mathrm{Hb} 7 \mathrm{~g} / \mathrm{dl}$ to a target of 7-9 g/dl), with the complications rate found to be higher in the liberal transfusion group [156] (level 1). A number of studies have reported higher mortality rates in liberal transfusion groups [157] (level 1). According to these studies, blood transfusion should be initiated at $\mathrm{Hb} 7 \mathrm{~g} / \mathrm{dl}$ with a target of 7-9 $\mathrm{g} / \mathrm{dl}$; however, the route and compositions of infusions should be carefully considered in the acute phase of acute abdomen $[150,151]$ (level 1).

CQ105 Which analgesic should be used for abdominal pain due to acute abdomen?

Regardless of cause, early analgesic use before a definitive diagnosis is recommended.
Intravenous administration of $1,000 \mathrm{mg}$ acetaminophen is recommended regardless of pain severity (level 1, recommendation A).

Intravenous narcotic analgesics should be added according to pain severity.

Anticonvulsants, such as butylscopolamine bromide, may be used as an adjuvant therapy for colic rather than the first drug of choice for abdominal pain (level 1, recommendation A) (Fig. 3).

Morphine, opioids such as fentanyl (level 1, recommendation A), an antagonistic analgesic such as pentazocine, and buprenorphine (level 2, recommendation A) should be considered in cases of acute abdomen (CQ92).

NSAIDs are as effective as opioids for colic of the biliary tract and may become the first drug of choice (level 1, recommendation $\mathrm{B}$ ).

NSAIDs should be used in cases of colic due to ureteral stones. When NSAIDs cannot be used, opioids are recommended (level 1, recommendation A). 
Table 19 Differential diagnosis in the elderly patients with acute abdomen

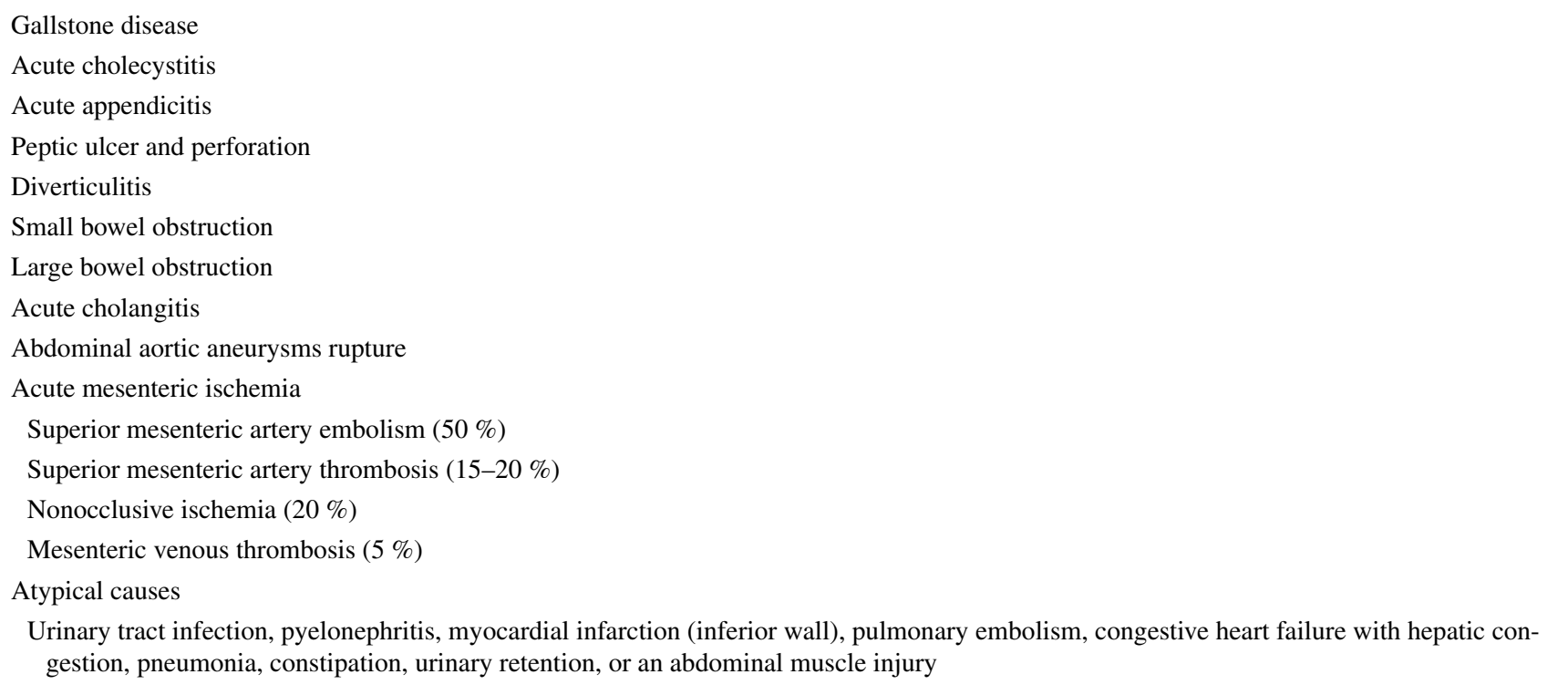

Modified from reference [147]

Table 20 Key clinical recommendations for elderly patients with acute abdomen

Consider cholecystitis even if an older patient does not present with classic symptoms, because they often are absent in older persons

Consider small bowel obstruction in the older patient with a history of surgery who presents with diffuse, colicky pain, nausea, vomiting, altered bowel sounds, distention, dehydration, diffuse tenderness, and possibly an ill-defined mass

Consider abdominal aortic aneurysm in the older patient with back or abdominal pain, particularly if they are male or have a history of tobacco use

Consider acute mesenteric ischemia if a patient presents with severe, poorly localized pain out of proportion to physical findings

Reference [147]

$<$ Comment $>$ According to a systematic review regarding the use of analgesics in adults presenting with acute abdomen to the emergency room, early usage of analgesics was associated with improved diagnosis and treatment. In this review, early analgesic use was recommended before a definite diagnosis, regardless of underlying cause (CQ92) [158-161]. Good control of analgesia use is recommended according to the strength and property of reported pain (Fig. 3) [161] (level 1).

CQ106 When should antimicrobial agents be given for acute abdomen?

When abdominal infections are diagnosed or suspected, blood cultures should be taken and antimicrobial agents administered (level 3, recommendation A).

In cases of septic shock due to infected acute abdomen, antibiotics should be initiated within $1 \mathrm{~h}$ of presentation (level 3, recommendation A).

When surgery is performed, additional antimicrobial agents should be administered just prior to surgery to prevent surgical site infection (level 2, recommendation A).

$<$ Comment> In patients with septic shock including intra-abdominal infections, delayed administration of antimicrobial agent has been shown to be correlated with an increased mortality [162, 163] (level 3). Based on these studies and sepsis guidelines (SSCG 2012), the administration of antimicrobial agents is recommended within $1 \mathrm{~h}$ of presentation after blood cultures sampling in patients with septic shock [150, 151] (level 3). The complicated intraabdominal infections guidelines (SIS and IDSA guidelines) recommended antimicrobial agents should be given when intra-abdominal infections are diagnosed or suspected in cases without shock within $8 \mathrm{~h}$ after presentation or in septic shock while in the emergency room [149] (level 5). When surgery for the intra-abdominal infections is necessary, antimicrobial agents should be given for less than $1 \mathrm{~h}$ (if possible, less than $30 \mathrm{~min}$ ) prior to the start of surgery. Maintaining the perioperative therapeutic range of antibiotics significantly reduced the risk of surgical site infection [164, 165] (level 2). 
Fig. 3 Algorithm for the selection of prediagnostic analgesia for acute abdominal pain in the general adult population. Reference [162]

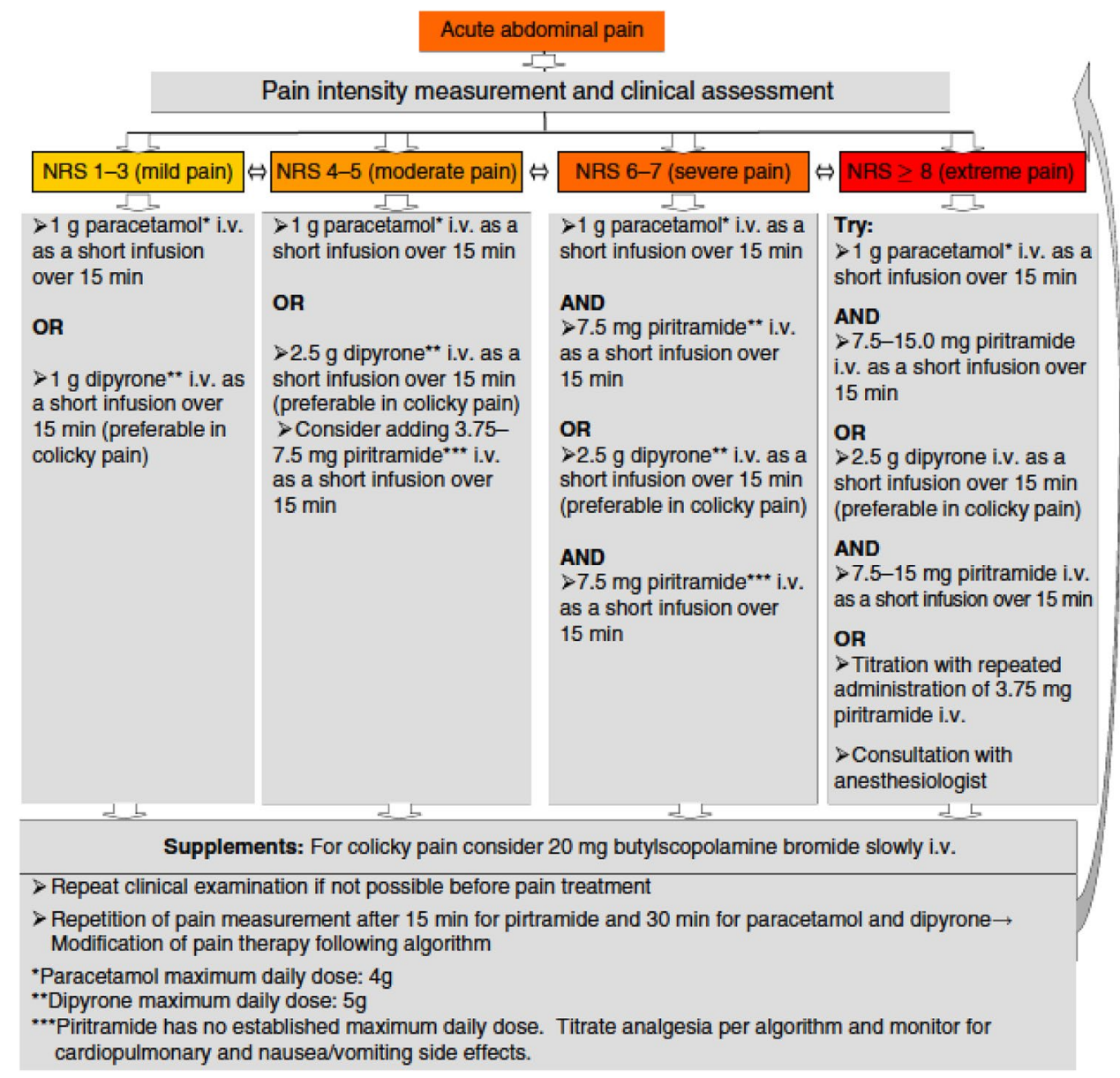

\subsection{Education (Table 10)}

CQ107 Are there educational programs that improve practitioner's skill in diagnosing and managing acute abdomen?

A meta-analysis of educational and computer-assisted diagnoses programs aimed at the improvement of practice skill regarding acute abdomen demonstrated improvements in diagnostic precision and decision making (level 3, recommendation $\mathrm{C} 1$ ).

$<$ Comment $>$ Methods of support for inexperienced staff in the diagnosis and management of patients with acute abdominal pain were prospectively evaluated by a previously reported study. (a) Structured data collection forms, (b) real-time computer-aided decision support, and (c) computer-based teaching packages were compared. The use of any one modality resulted in improved diagnostic accuracy and decision-making performance [166] (level 3).

In a meta-analysis of the efficacy of a computer-assisted systems in improving decision making in cases of acute abdominal pain, diagnostic precision was improved by $17.25 \%$ [167] (level 1).
A previous study reported the use of a trans-vaginal echo training program for emergency residents in improving the detection of extrauterine pregnancy. After the educational program, the rate of agreement in findings among attending physicians was $93.3 \%$, and first-year residents were found to have a higher rate of agreement than 2 and 3 years, indicating the importance of early training [168] (level 3).

As described above, educational programs taking acute abdomen into consideration have been developed and shown to improve clinical practice skills.

CQ108 Is the algorithm for the management of acute abdomen based on current evidence?

Although there is a practice algorithm for the management of childbearing women, the algorithm has been shown to have utility in the exclusion of acute appendicitis, AAA rupture, and extrauterine pregnancy by triage nurses. Further, a computer-aided diagnostic program has been reported; however, the efficacy of the practice algorithm in improving the prognosis of acute abdomen has yet to be validated (level 5, recommendation $\mathrm{C} 1$ ). 
$<$ Comment> An initial algorithm for the management of childbearing women and patients with other acute abdomen etiologies by physicians has been developed [169] (level 5). In childbearing women, the algorithm excludes peritonitis, extrauterine pregnancy, adnexitis, and pelvic inflammatory disease, and the plain algorithm excludes peritonitis, AAA, acute coronary syndrome, intestinal ischemia, and bowel obstruction.

An exclusion algorithm for triage nurses has also been introduced that allows the exclusion of acute appendicitis, AAA rupture, and extrauterine pregnancy. For adults presenting with acute abdominal pain, medical practitioners should take a detailed medical history, perform a brief medical examination, measure urinary hCG in childbearing women and pay maximum attention to women and elderly people due to a substantially higher misdiagnosis in these populations [170] (level 5).

Using the advanced triage algorithm for acute abdominal pain approved by physicians, triage nurses can shorten sojourn times in the emergency room to an average of 46 min [171] (level 4).

\section{Limitations}

The Practice Guidelines for Primary Care of Acute Abdomen 2015 were made considering the characteristics of the Japanese medical system such as easy access to hospitals and the ready availability of ultrasonography, MDCT, and MRI in emergency rooms. Therefore, some recommendations may not be applicable to other countries with different medical systems compared with those of Japan.

Moreover, because these guidelines are the first guidelines for acute abdomen, they need to be validated, revised, and updated in clinical practice based on feedbacks and new evidences.

\section{Conclusion}

The Practice Guidelines for Primary Care of Acute Abdomen 2015 have been prepared as the first evidence-based guidelines for the evaluation and management of acute abdomen. It is hoped that these guidelines will contribute to improving physician practice, primary care, and the prognosis of patients with acute abdomen.

\section{Compliance with ethical standards}

Conflict of interest The authors have no financial support or "Conflict of Interest" to declare. Prior to the preparation of these medical guidelines, all members of the Guidelines Revision Committee declared any conflicts of interest. Effort was made to avoid biases in the guidelines with regard to economic issues. Effort was also made to create a cooperative system with a number of relevant academic societies and research organizations to avoid academic conflicts of interest among individual academic societies.

Economic independence All of the expenses incurred in the preparation and publication of these guidelines were paid for by the relevant academic societies. No funding or grants were received from pharmaceutical or other companies.

\section{References}

1. OCEBM Levels of Evidence Working Group. The Oxford 2011 Levels of Evidence. Oxford Centre for Evidence-Based Medicine. http://www.cebm.net/index.aspx?o=5653.

2. Jaeschke R, Guyatt GH, Dellinger P, Schünemann H, Levy MM, Kunz R, et al. GRADE working group. Use of GRADE grid to reach decisions on clinical practice guidelines when consensus is elusive. BMJ. 2008;337:a744.

3. Guyatt G, Oxman AD, Akl EA, Kunz R, Vist G, Brozek J, et al. GRADE guidelines: 1. Introduction-GRADE evidence profiles and summary of findings tables. J Clin Epidemiol. 2011;64:383-94.

4. Guyatt GH, Oxman AD, Kunz R, Atkins D, Brozek J, Vist G, et al. GRADE guidelines: 2. Framing the question and deciding on important outcomes. J Clin Epidemiol. 2011;64:395-400.

5. Balshem H, Helfand M, Schünemann HJ, Oxman AD, Kunz $\mathrm{R}$, Brozek J, et al. GRADE guidelines: 3 . Rating the quality of evidence consensus is elusive. BMJ 2008. J Clin Epidemiol. 2011;64:401-6.

6. Guyatt GH, Oxman AD, Vist G, Kunz R, Brozek J, AlonsoCoello P, et al. GRADE guidelines: 4 . Rating the quality of evidence-study limitations (risk of bias). J Clin Epidemiol. 2011;64:407-15.

7. Guyatt GH, Oxman AD, Montori V, Vist G, Kunz R, Brozek J, et al. GRADE guidelines: 5. Rating the quality of evidencepublication bias. J Clin Epidemiol. 2011;64:1277-82.

8. Guyatt GH, Oxman AD, Kunz R, Brozek J, Alonso-Coello P, Rind D, et al. GRADE guidelines 6 . Rating the quality of evidence-imprecision. J Clin Epidemiol. 2011;64:1283-93.

9. Guyatt GH, Oxman AD, Kunz R, Woodcock J, Brozek J, Helfand M, et al. GRADE Working Group. GRADE guidelines: 7. Rating the quality of evidence-inconsistency. J Clin Epidemiol. 2011;64:1294-302.

10. Guyatt GH, Oxman AD, Kunz R, Woodcock J, Brozek J, Helfand M, et al. GRADE Working Group. GRADE guidelines: 8. Rating the quality of evidence-indirectness. J Clin Epidemiol. 2011;64:1303-10.

11. Guyatt GH, Oxman AD, Sultan S, Glasziou P, Akl EA, AlonsoCoello $P$, et al. GRADE Working Group. GRADE guidelines: 9. Rating up the quality of evidence. J Clin Epidemiol. 2011;64:1311-6.

12. Brunetti M, Shemilt I, Pregno S, Vale L, Oxman AD, Lord J, et al. GRADE guidelines: 10. Considering resource use and rating the quality of economic evidence. J Clin Epidemiol. 2013;66:140-50.

13. Guyatt G, Oxman AD, Sultan S, Brozek J, Glasziou P, AlonsoCoello P, et al. GRADE guidelines: 11. Making an overall rating of confidence in effect estimates for a single outcome and for all outcomes. J Clin Epidemiol. 2013;66:151-7.

14. Guyatt GH, Oxman AD, Santesso N, Helfand M, Vist G, Kunz $\mathrm{R}$, et al. GRADE guidelines: 12. Preparing summary of findings tables-binary outcomes. J Clin Epidemiol. 2013;66:158-72. 
15. Guyatt GH, Thorlund K, Oxman AD, Walter SD, Patrick D, Furukawa TA, et al. GRADE guidelines: 13. Preparing summary of findings tables and evidence profiles-continuous outcomes. J Clin Epidemiol. 2013;66:173-83.

16. Andrews J, Guyatt G, Oxman AD, Alderson P, Dahm P, FalckYtter Y, et al. GRADE guidelines: 14. Going from evidence to recommendations: the significance and presentation of recommendations. J Clin Epidemiol. 2013;66:719-25.

17. Andrews J, Guyatt G, Oxman AD, Pottie K, Meerpohl JJ, Coello PA, et al. GRADE guidelines: 15. Going from evidence to recommendations-determinants of a recommendation's direction and strength. J Clin Epidemiol. 2013;66:726-35.

18. Takada T, Hirata K, Mayumi T, Yoshida M, Sekimoto M, Hirota $\mathrm{M}$, et al. Cutting-edge information for the management of acute pancreatitis. J Hepatobiliary Pancreat Sci. 2010;17:3-12.

19. Silen W. Cope's early diagnosis of the acute abdomen. 22nd ed. New York: Oxford University Press; 2010 (level 5).

20. Powers RD, Guertler AT. Abdominal pain in the ED: stability and change over 20 years. Am J Emerg Med. 1995;13:301-3 (level 4).

21. Cartwright SL, Knudson MP. Evaluation of acute abdominal pain in adults. Am Fam Physician. 2008;77:971-8 (level 4).

22. Lanas A, García-Rodríguez LA, Polo-Tomás M, et al. Time trends and impact of upper and lower gastrointestinal bleeding and perforation in clinical practice. Am J Gastroenterol. 2009;104:1633-41 (level 2).

23. Ince M, Stocchi L, Khomvilai S, et al. Morbidity and mortality of the Hartmann procedure for diverticular disease over 18 years in a single institution. Colorectal Dis. 2012;14:e492-8 (level 3).

24. Konno H, Wakabayashi G, Udagawa H, et al. Annual Report of National Clinical Database in Gastroenterological Surgery 2011-2012. Jpn J Gastroenterol Surg. 2013;46:952-63 (in Japanese) (level 2) .

25. Telfer S, Fenyö G, Holt PR, et al. Acute abdominal pain in patients over 50 years of age. Scand J Gastroenterol Suppl. 1988;144:47-50 (level 3).

26. Kriwanek S, Armbruster C, Beckerhinn P, et al. Prognostic factors for survival in colonic perforation. Int $\mathrm{J}$ Colorectal Dis. 1994;9:158-62 (level 3).

27. Styrud J, Eriksson S, Granström L. Treatment of perforated appendicitis: an analysis of 362 patients treated during 8 years. Dig Surg. 1998;15:683-6 (level 3).

28. Gajic O, Urrutia LE, Sewani H, et al. Acute abdomen in the medical intensive care unit. Crit Care Med. 2002;30:1187-90 (level 3).

29. Kettunen J, Paajanen H, Kostiainen S. Emergency abdominal surgery in the elderly. Hepatogastroenterology. 1995;42:106-8 (level 3).

30. Biondo S, Ramos E, Deiros M, et al. Prognostic factors for mortality in left colonic peritonitis: a new scoring system. J Am Coll Surg. 2000;191:635-42 (level 3).

31. Arenal JJ, Bengoechea-Beeby M. Mortality associated with emergency abdominal surgery in the elderly. Can J Surg. 2003;46:111-6 (level 3).

32. Kawai K, Hiramatsu T, Kobayashi R, et al. Coagulation disorder as a prognostic factor for patients with colorectal perforation. J Gastroenterol. 2007;42:450-5 (level 3).

33. Simić $\mathrm{O}$, Strathausen $\mathrm{S}$, Hess $\mathrm{W}$, et al. Incidence and prognosis of abdominal complications after cardiopulmonary bypass. Cardiovasc Surg. 1999;7:419-24 (level 3).

34. van Geloven AA, Biesheuvel TH, Luitse JS, et al. Hospital admissions of patients aged over 80 with acute abdominal complaints. Eur J Surg. 2000;166:866-71 (level 3).

35. Dimick JB, Stanley JC, Axelrod DA, et al. Variation in death rate after abdominal aortic aneurysmectomy in the United
States: impact of hospital volume, gender, and age. Ann Surg. 2002;235:579-85 (level 2).

36. Limmer DJ, O'Keefe MF, Grant HT, et al. Emergency Care. 11th ed. Englewood Cliffs: N.J., Prentice Hall; 2008. p. 242 (level 4).

37. Thomas SA. Spinal stenosis: history and physical examination. Phys Med Rehabil Clin N Am. 2003;14:29-39 (level 4).

38. Fishman MB, Aronson MD, History and physical examination in adults with abdominal pain. UpToDate, this topic last up, 2014. (level 4).

39. Hernández-Díaz S, Rodríguez LA. Association between nonsteroidal anti-inflammatory drugs and upper gastrointestinal tract bleeding/perforation an overview of epidemiologic studies published in the 1990s. Arch Int Med. 2000;160:2093-9 (level 1).

40. Fadul CE, Lemann W, Thaler HT, et al. Perforation of the gastrointestinal tract in patients receiving steroids for neurologic disease. Neurology. 1988;38:348-52 (level 3).

41. Marshall LF, King J, Langfitt TW. The complications of highdose corticosteroid therapy in neurosurgical patients: a prospective study. Ann Neurol. 1977;1:201-3 (level 3).

42. Cartwright SL, Knudson MP. Evaluation of acute abdominal pain in adults. Am Fam Physician. 2008;77:971-8 (level 5).

43. Gray DW, Dixon JM, Collin J. The closed eyes sign: an aid to diagnosing non-specific abdominal pain. BMJ. 1988;297:837 (level 2).

44. Van den Bruel A, Thompson M, Buntinx F, et al. Clinicians' gut feeling about serious infections in children: observational study. BMJ. 2012;345:e6144 (level 3).

45. Parker MM, Shelhamer JH, Natanson C, et al. Serial cardiovascular variables in survivors and nonsurvivors of human septic shock: heart rate as an early predictor of prognosis. Crit Care Med. 1987;15:923-9 (level 4).

46. Arnell TD, de Virgilio C, Chang L, et al. Admission factors can predict the need for ICU monitoring in gallstone pancreatitis. Am Surg. 1996;62:815-9 (level 3).

47. Lemeshow S, Teres D, Klar J, et al. Mortality Probability Models(MPM II)based on an international cohort of intensive care unit patients. JAMA. 1993;270:2478-86 (level 3).

48. Shapiro NI, Wolfe RE, Moore RB, et al. Mortality in Emergency Department Sepsis(MEDS) score: a prospectively derived and validated clinical prediction rule. Crit Care Med. 2003;31:670-5 (level 3).

49. Vales EC, Abraira V, Sánchez JC, et al. A predictive model for mortality of bloodstream infections. Bedside analysis with the Weibull function. J Clin Epidemiol. 2002;55:563-72 (level 3).

50. McGee S, Abernethy WB 3rd, Simel DL. The rational clinical examination. Is this patient hypovolemic? JAMA. 1999;281:1022-9 (level 2).

51. McGee S. Evidence-based physical diagnosis. 3rd ed. Philadelphia: Saunders; 2012 (level 2).

52. Pittet D, Thiévent B, Wenzel RP, et al. Bedside prediction of mortality from bacteremic sepsis. A dynamic analysis of ICU patients. Am J Respir Crit Care Med. 1996;153:684-93 (level 3).

53. Howie CR, Gunn AA. Temperature: a poor diagnostic indicator in abdominal pain. J R Coll Surg Edinb. 1984;29:249-51 (level 3).

54. Clinical policy: critical issues for the initial evaluation and management of patients presenting with a chief complaint of nontraumatic acute abdominal pain. Ann Emerg Med 2000; 36: 406-15 (level 5).

55. Hoffmann J, Kurgan A, Abramowitz HB. Rectal temperature in the diagnosis of acute lower abdominal pain. Isr J Med Sci. 1983;19:505-7 (level 4).

56. Parker JS, Vukov LF, Wollan PC. Abdominal pain in the elderly: use of temperature and laboratory testing to screen for surgical disease. Fam Med. 1996;28:193-7 (level 4). 
57. Baid H. A Critical review of auscultating bowel sounds. Br J Nurs. 2009; 18:1125-9 (level 2).

58. Mangione S. Physical diagnosis secrets. 2nd ed. Philadelphia: Mosby; 2007 (level 5).

59. Eskelinen M, Ikonen J, Lipponen P. Contributions of historytaking, physical examination, and computer assistance to diagnosis of acute small-bowel obstruction. A prospective study of 1333 patients with acute abdominal pain. Scand J Gastroenterol. 1994;29:715-21 (level 3).

60. Ching SS, Tan YK. Spectral analysis of bowel sounds in intestinal obstruction using an electronic stethoscope. World J Gastroenterol 2012; 18: 4585-92. PM 22969233. (level 4).

61. Arnbjörnsson E. Normal and pathological bowel sound patterns. Ann Chir Gynaecol. 1986;75:314-8 (level 4).

62. Durup-Dickenson M, Christensen MK, Gade J. Abdominal auscultation does not provide clear clinical diagnoses. Dan Med J. 2013;60:A4620 (level 3).

63. Gade J, Kruse P, Andersen OT, et al. Physicians' Abdominal Auscultation. Scand J Gastroenterol. 1998;33:773-7 (level 3).

64. Gu Y, Lim HJ, Moser MA. How useful are bowel sounds in assessing the abdomen? Dig Surg. 2010;27:422-6 (level 3).

65. Turnbull JM. The rational clinical examination. Is listening for abdominal bruits useful in the evaluation of hypertension. JAMA. 1995;274:1299-301 (level 2).

66. Williams JW, Simel DL. The rational clinical examination. Does this patient have ascites? How to divine fluid in the abdomen. JAMA. 1992;267:2645-8 (level 2).

67. Lederle FA, Simel DL. The rational clinical examination. Does this patient have abdominal aortic aneurysm? JAMA. 1999;281:77-82 (level 2).

68. Trowbridge RL, Rutkowski NK, Shojania KG. Does this patient have acute cholecystitis? JAMA. 2003;289:80-6 (level 2).

69. Wagner JM, Mckinney WP, Carpenter JL. Does this patient have appendicitis? JAMA. 1996;276:1589-94 (level 2).

70. Andersson RE. Meta-analysis of the clinical and laboratory diagnosis of appendicitis. Br J Surg. 2004;91:28-37 (level 2).

71. Fenyo G. Acute abdominal disease in the elderly: experience from two series in Stockholm. Am J Surg. 1982;143:751-4 (level 4).

72. Andeweg CS, Knobben L, Hendriks JC, et al. How to diagnose acute left-sided colonic diverticulitis. Ann Surg. 2011;253:940-6 (level 3).

73. Corsetti JP, Arvan DA. Acute pancreatitis. In: Black ER, Bordley DR, Tape TG, Panzer RJ, editors. Diagnostic strategies for common medical problems. 2nd ed. Philadelphia: American College of Physicians; 1999. p. 205 (level 4).

74. Cudnik MT, Darbha S, Jones J, et al. The diagnosis of acute mesenteric ischemia: a systematic review and meta-analysis. Acad Emerg Med. 2013;20:1087-100 (level 2).

75. Carnett JB. Intercostal neuralgia as a cause of abdominal pain and tenderness. J Surg Gynecol Obstet. 1926;42:625-32 (level 5).

76. Thomson H, Francis DM. Abdominal-wall tenderness. Lancet. 1977;2:1053-4 (level 3).

77. Kawsar HI, Shahnewaz J, Bhansali H, et al. Rectus sheath haematoma. BMJ Case Reports. 2012. doi:10.1136/ bcr.02.2012.5909 (level 5).

78. Srinivasan R, Greenbaum DS. Chronic abdominal wall pain: a frequently overlooked problem. Practical approach to diagnosis and management. Am J Gastroenterol. 2002;97:824-30 (level 5).

79. Panebianco NL, Jahnes K, Mills AM. Imaging and laboratory testing in acute abdominal pain. Emerg Med Clin North Am. 2011;29:175-93 (level 5).

80. Pitts SR, Niska RW, Xu J, et al. National Hospital Ambulatory Medical Care Survey: 2006 emergency department summary. Natl Health Stat Report. 2008;6:1-38 (level 3).
81. Nagurney JT, Brown DF, Chang Y, et al. Use of diagnostic testing in the emergency department for patients presenting with non traumatic abdominal pain. J Emerg Med. 2003;25:363-71 (level 3).

82. Kafetzis DA, Velissariou IM, Nikolaides P, et al. Procalcitonin as a predictor of severe appendicitis in children. Eur $\mathbf{J}$ Clin Microbiol Infect Dis. 2005;24:484-7 (level 3).

83. Sand M, Trullen XV, Bechara FG, et al. A prospective bicenter study investigating the diagnostic value of procalcitonin in patients with acute appendicitis European surgical research. Europaische chirurgische Forschung. Recherches chirurgicales europeennes. 2009;43:291-7 (level 3).

84. Anielski R, Kusnierz-Cabala B, Szafraniec K. An evaluation of the utility of additional tests in the preoperative diagnostics of acute appendicitis Langenbeck's archives of surgery/Deutsche Gesellschaft für Chirurgie. 2010;395:1061-8 (level 3).

85. Chandel V, Batt SH, Bhat MY, et al. Procalcitonin as the biomarker of inflammation in diagnosis of appendicitis in pediatric patients and prevention of unnecessary appendectomies. Indian J Surg. 2011;73:136-41 (level 4).

86. Wu JY, Chen HC, Lee SH, et al. Diagnostic role of procalcitonin in patients with suspected appendicitis. World J Surg. 2012;36:1744-9 (level 3).

87. Kaya B, Sana B, Eris C, et al. The diagnostic value of D-dimer, procalcitonin and CRP in acute appendicitis. Int $\mathrm{J}$ Med Sci. 2012;9:909-15 (level 4).

88. Yu CW, Juan LI, Wu MH, et al. Systematic review and metaanalysis of the diagnostic accuracy of procalcitonin, C-reactive protein and white blood cell count for suspected acute appendicitis. Br J Surg. 2013;100:322-9 (level 1).

89. Mofidi R, Suttie SA, Patil PV, et al. The value of procalcitonin at predicting the severity of acute pancreatitis and development of infected pancreatic necrosis: systematic review. Surgery. 2009;146:72-81 (level 1).

90. Kruse O, Grunnet N, Barfod C. Blood lactate as a predictor for in-hospital mortality in patients admitted acutely to hospital: a systematic review. Scand J Trauma Resusc Emerg Med. 2011;19:74 (level 1).

91. Acosta S, Nilsson T. Current status on plasma biomarkers for acute mesenteric ischemia. J Thromb Thrombolysis. 2012;33:355-61 (level 2).

92. Lavery RF, Livingston DH, Tortella BJ, et al. The utility of venous lactate to triage injured patients in the trauma center. $\mathrm{J}$ Am Coll Surg. 2000;190:656-64 (level 4).

93. Younger JG, Falk JL, Rothrock SG. Relationship between arterial and peripheral venous lactate levels. Acad Emerg Med. 1996;3:730-4 (level 3).

94. Gallagher EJ, Rodriguez K, Touger M. Agreement between peripheral venous and arterial lactate levels. Ann Emerg Med. 1997;29:479-83 (level 3).

95. Medina-Bombardo D, Jover-Palmer A. Does clinical examination aid in the diagnosis of urinary tract infections in women? A systematic review and meta-analysis. BMC Fam Pract. 2011:12:111 (level 1).

96. Mody L, Juthani-Mehta M. Urinary tract infections in older women: a clinical review. JAMA. 2014;311:844-54 (level 2).

97. Arora S, Henderson SO, Long T, et al. Diagnostic accuracy of point-of-care testing for diabetic ketoacidosis at emergencydepartment triage: $\beta$-hydroxybutyrate versus the urine dipstick. Diabetes Care. 2011;34:852-4 (level 3).

98. Hendey GW, Schwab T, Soliz T. Urine ketone dip test as a screen for ketonemia in diabetic ketoacidosis and ketosis in the emergency department. Ann Emerg Med. 1997;29:735-8 (level 4).

99. Fann JI, Sarris GE, Mitchell RS, et al. Treatment of patients with aortic dissection presenting with peripheral vascular complications. Ann Surg. 1990;212:705-13 (level 4). 
100. Eisenberg RL, Heineken P, Hedgcock MW, et al. Evaluation of plain abdominal radiographs in the diagnosis of abdominal pain. Ann Surg. 1983;197:464-9 (level 2).

101. Mirvis SE, Young JW, Keramati B, et al. Plain film evaluation of patients with abdominal pain: are three radiographs necessary? Am J Roentgenol. 1986;147:501-3 (level 3).

102. Anyanwu AC, Moalypour SM. Are abdominal radiographs still overutilized in the assessment of acute abdominal pain? A district general hospital audit. J R Coll Surg Edinb. 1998;43:26770 (level 3)

103. Kellow ZS, MacInnes M, Kurzencwyg D, et al. The role of abdominal radiography in the evaluation of the nontrauma emergency patient. Radiology. 2008;248:887-93 (level 3).

104. Stower MJ, Amar SS, Mikulin T, et al. Evaluation of the plain abdominal X-ray in the acute abdomen. J R Soc Med. 1985;78:630-3 (level 3).

105. MacKersie AB, Lane MJ, Gerhardt RT, et al. Nontraumatic acute abdominal pain: unenhanced helical CT compared with three-view acute abdominal series. Radiology. 2005;237:11422 (level 3)

106. Lameris W, van Randen A, van Es HW, et al. Imaging strategies for detection of urgent conditions in patients with acute abdominal pain: diagnostic accuracy study. BMJ. 2009;338:b2431 (level 2).

107. Ahn SH, Mayo-Smith WW, Murphy BL, et al. Acute nontraumatic abdominal pain in adult patients: abdominal radiography compared with CT evaluation. Radiology. 2002;225:159-64 (level 3).

108. Miller RE, Nelson SW. The roentgenologic demonstration of tiny amounts of free intraperitoneal gas: experimental and clinical studies. Am J Roentgenol. 1971;112:574-85 (level 4).

109. Silen W. Abdominal pain. In: Thom GW, Braunwald E, Isselbacher KJ, et al., editors. Harrison's principles of internal medicine. New York: McGraw-Hill; 1975. p. 33-7 (level 5).

110. Lindelius A, Torngren S, Sonden A, et al. Impact of surgeonperformed ultrasound on diagnosis of abdominal pain. Emerg Med J. 2008;25:486-91 (level 2).

111. Lindelius A, Torngren S, Pettersson H, et al. Role of surgeonperformed ultrasound on further management of patients with acute abdominal pain: a randomised controlled clinical trial. Emerg Med J. 2009;26:561-6 (level 2).

112. Allemann F, Cassina P, Röthlin M, et al. Ultrasound scans done by surgeons for patients with acute abdominal pain: a prospective study. Eur J Surg. 1999;165:966-70 (level 2).

113. Mazzei MA, Guerrini S, Cioffi Squitieri N, et al. The role of US examination in the management of acute abdomen. Crit Ultrasound J 2013; 5 Suppl 1: S6. PM 23902801 (level 4).

114. Stoker J, van Randen A, Lameris W, et al. Imaging patients with acute abdominal pain. Radiology. 2009;253:31-46 (level 5).

115. Harvey RT, Miller WT Jr. Acute biliary disease: initial CT and follow-up US versus initial US and follow-up CT. Radiology. 1999;213:831-6 (level 3).

116. O'Malley ME, Wilson SR. Ultrasonography and computed tomography of appendicitis and diverticulitis. Semin Roentgenol. 2001;36:138-47 (level 3).

117. van Randen A, Lameris W, van Es HW, et al. A comparison of the accuracy of ultrasound and computed tomography in common diagnoses causing acute abdominal pain. Eur Radiol. 2011;21:1535-45 (level 3).

118. Laing FC. Ultrasonography of the acute abdomen. Radiol Clin North Am. 1992;30:389-404 (level 5).

119. Hudson PA, Promes SB. Abdominal ultrasonography. Emerg Med Clin North Am. 1997;15:825-48 (level 5).

120. Grassi R, Romano S, Pinto A, et al. Gastro-duodenal perforations: conventional plain film, US and CT findings in 166 consecutive patients. Eur J Radiol. 2004;50:30-6 (level 5).
121. Langer JE. Computed tomography and ultrasonography of acute renal abnormalities. Semin Roentgenol. 2001;36:99-107 (level 5).

122. Puylaert JB. Ultrasonography of the acute abdomen: gastrointestinal conditions. Radiol Clin N Am. 2003;41:1227-42 (level 5).

123. Noble VE, Nelson BP. Manual of Emergency and Critical Care Ultrasound. 2nd ed. Cambridge: Cambridge University Press; 2011.

124. Rosen MP, Sands DZ, Longmaid HE 3rd, et al. Impact of abdominal $\mathrm{CT}$ on the management of patients presenting to the emergency department with acute abdominal pain. Am J Roentgenol. 2000;174:1391-6 (level 2).

125. MacKersie AB, Lane MJ, Gerhardt RT, et al. Nontraumatic acute abdominal pain: unenhanced helical CT compared with three-view acute abdominal series. Radiology. 2005;237:114 22 (level 2).

126. Siewert B, Raptopoulos V, Mueller MF, et al. Impact of $\mathrm{CT}$ on diagnosis and management of acute abdomen in patients initially treated without surgery. Am J Roentgenol. 1997;168:173-8 (level 3).

127. Millet I, Alili C, Bouic-Pages E, et al. Acute abdominal pain in elderly patients: effect of radiologist awareness of clinicobiologic information on CT Accuracy. Am J Roentgenol. 2013;201:1171-9 (level 3).

128. Berrington de González A, Darby S. Risk of cancer from diagnostic X-rays: estimates for the UK and 14 other countries. Lancet. 2004;363:345-51 (level 4).

129. Mun S, Ernst RD, Chen K, et al. Rapid CT diagnosis of acute appendicitis with IV contrast material. Emerg Radiol. 2006;12:99-102 (level 3).

130. Tack D, Sourtzis S, Delpierre I, et al. Low-dose unenhanced multidetector CT of patients with suspected renal colic. Am J Roentgenol. 2003;180:305-11 (level 3).

131. Neitlich JD, Topazian M, Smith RC, et al. Detection of choledocholithiasis: comparison of unenhanced helical CT and endoscopic retrograde cholangiopancreatography. Radiology. 1997;203:753-7 (level 3).

132. Arvanitakis M, Delhaye M, De Maertelaere V, et al. Computed tomography and magnetic resonance imaging in the assessment of acute pancreatitis. Gastroenterology. 2004;126:715-23 (level 3).

133. Sartelli M, Catena F, Ansaloni L, et al. World Complicated intra-abdominal infections in Europe: a comprehensive review of the CIAO study. World J Emerg Surg. 2012;7:36 (level 2).

134. Doria AS, Moineddin R, Kellenberger CJ, et al. US or CT for diagnosis of appendicitis in children and adults? a metaanalysis. Radiology. 2006;241:83-94 (level 3).

135. Japan Society of Obstetrics and Gynecology, Japan Association of Obstetricians and Gynecologists.The Guidelines Obstetrical Practice in Japan 2014, Japan Society of Obstetrics and Gynecology, Tokyo, 2014 (in Japanese) (level 5).

136. International Commission on Radiological Protection. Pregnancy and medical radiation. Publication 84. Ann ICRP 2000;30:1-43 (level 5).

137. Streffer C, Shore R, Konermann G, et al. Biological effects after prenatal irradiation (embryo and fetus). A report of the International Commission on Radiological Protection. Ann ICRP. 2003;33:5-206 (Committee report) (level 5).

138. Miller RW. Discussion: severe mental retardation and cancer among atomic bomb survivors exposed in utero. Teratology. 1999;59:234-5 (level 3).

139. Brenner D, Elliston CD, Hall EJ, et al. Estimated risks of radiation-induced fatal cancer from pediatric CT. Am J Roentgenol. 2001;176:289-96 (level 3).

140. Pearce MS, Salotti JA, Little MP, et al. Radiation exposure from CT scans in childhood and subsequent risk of leukaemia 
and brain tumours: a retrospective cohort study. Lancet. 2012;380:499-505 (level 2).

141. Frederrick HM. Acute abdominal pain. In: Feldman M, Friedman LS, Brandt LJ, editors. Sleisenger and Fordtran's Gastrointestinal and Liver Disease. 9th ed, Phyladelphia: Saunders; 2010. p. 151-62 (level 5).

142. Fields JM, Dean AJ. Systemic causes of abdominal pain. Emerg Med Clin North Am. 2011;29:195-210 (level 5).

143. Laurell H, Hansson LE, Gunnarsson U. Diagnostic pitfalls and accuracy of diagnosis in acute abdominal pain. Scand J Gastroenterol. 2006;41:1126-31 (level 3).

144. Esses D, Birnbaum A, Bijur P, et al. Ability of CT to alter decision making in elderly patients with acute abdominal pain. Am J Emerg Med. 2004;22:270-2 (level 3).

145. Hustey FM, Meldon SW, Banet GA, et al. The use of abdominal computed tomography in older ED patients with acute abdominal pain. Am J Emerg Med. 2005;23:259-65 (level 3).

146. Marco CA, Schoenfeld CN, Keyl PM, et al. Abdominal pain in geriatric emergency patients:variables associated with adverse outcomes. Acad Emerg Med. 1998;5:1163-8 (level 3).

147. Lyon C, Clark DC. Diagnosis of acute abdominal pain in older patients. Am Fam Physician. 2006;74:1537-44 (level 5).

148. Barne BA, Behringer GE, Wheelock FC, et al. Treatment of appendicitis at the Massachusetts General Hospital. JAMA. 1962;180:122-6 (level 3).

149. Solomkin JS, Mazuski JE, Bradley JS, et al. Diagnosis and management of complicated intra-abdominal infection in adults and children: guidelines by the Surgical Infection Society and the Infectious Diseases Society of America. Clin Infect Dis. 2010;50:133-64 (level 5).

150. Dellinger RP, Levy MM, Rhodes A, et al. Surviving Sepsis Campaign: international guidelines for management of severe sepsis and septic shock, 2012. Intensive Care Med. 2013;39:165-228 (level 1 5).

151. Dellinger RP, Levy MM, Rhodes A, et al. Surviving Sepsis Campaign: international guidelines for management of severe sepsis and septic shock: 2012. Crit Care Med. 2013;41:580-637 (level 1 5)

152. Finfer S, McEvoy S, Bellomo R, et al. Impact of albumin compared to saline on organ function and mortality of patients with severe sepsis. Intensive Care Med. 2011;37:86-96 (level 1).

153. Finfer S, Bellomo R, Boyce N, et al. A comparison of albumin and saline for fluid resuscitation in the intensive care unit. $\mathrm{N}$ Engl J Med. 2004;350:2247-56 (level 1).

154. Myburgh JA, Finfer S, Billot L. Hydroxyethyl starch or saline in intensive care. N Engl J Med. 2013;368:775 (level 1).

155. Perner A, Haase N, Guttormsen AB, et al. Hydroxyethyl starch 130/0.42 versus Ringer's acetate in severe sepsis. N Engl $\mathbf{J}$ Med. 2012;367:124-34 (level 1).

156. Hebert PC, Wells G, Blajchman MA, et al. A multicenter, randomized, controlled clinical trial of transfusion requirements in critical care. Transfusion Requirements in Critical Care Investigators, Canadian Critical Care Trials Group. N Engl J Med. 1999;340:409-17 (level 1).

157. Vincent JL, Baron JF, Reinhart K, et al. Anemia and blood transfusion in critically ill patients. JAMA. 2002;288:1499-507 (level 1).
158. Manterola C, Vial M, Moraga J, et al. Analgesia in patients with acute abdominal pain. Cochrane Database of Systematic Reviews 2011;1 (level 1).

159. Oguzturk H, Ozgur D, Turtay MG, et al. Tramadol or paracetamol do not effect the diagnostic accuracy of acute abdominal pain with significant pain relief-a prospective, randomized, placebo controlled double blind study. Eur Rev Med Pharmacol Sci. 2012;16:1983-8 (level 2).

160. Savoia G, Coluzzi F, Di Maria C, et al. Italian Intersociety Recommendations(SIAARTI, SIMEU, SIS 118, AISD, SIARED, SICUT, IRC)on Pain Management in the Emergency Setting. Minerva Anestesiol 2014 May 22. (level 5).

161. Falch C, Vicente D, Häberle H, et al. Treatment of acute abdominal pain in the emergency room: a systemati c review of the literature. Eur J Pain 2014 (level 5).

162. Kumar A, Roberts D, Wood KE, et al. Duration of hypotension before initiation of effective antimicrobial therapy is the critical determinant of survival in human septic shock. Crit Care Med. 2006;34:1589-96 (level 3).

163. Puskarich MA, Trzeciak S, Shapiro NI, et al. Association between timing of antibiotic admission and mortality from septic shock in patients treated with a quantitative resuscitation protocol. Crit Care Med. 2011;39:2066-71 (level 3).

164. Bratzler DW, Houck PM. Antimicrobial prophylaxis for surgery:an advisory statement from National Surgical Infection Prevention Project. Am J Surg. 2005;189:395-404 (level 2).

165. Bratzler DW, Hunt DR. The surgical infection prevention and surgical care improvement projects: national initiatives to improve outcomes for patients having surgery. Clin Infect Dis. 2006;43:322-30 (level 2).

166. de Dombal FT, Dallos V, McAdam WA. Can computer aided teaching packages improve clinical care in patients with acute abdominal pain? BMJ. 1991;302:1495-7 (level 3).

167. Cooper JG, West RM, Clamp SE, et al. Does computeraided clinical decision support improve the management of acute abdominal pain? A systematic review. Emerg Med J. 2011;28:553-7 (level 1).

168. MacVane CZ, Irish CB, Strout TD, et al. Implementation of transvaginal ultrasound in an emergency department residency program:an analysis of resident interpretation. J Emerg Med. 2012;43:124-8 (level 3).

169. Kendall JL, Moreira ME. Evaluation of the adult with abdominal pain in the emergency department. UpToDate (level 5).

170. Dagiely S. An algorithm for triaging commonly missed causes of acute abdominal pain. J Emerg Nurs. 2006;32:91-3 (level 5).

171. Cheung WW, Heeney L, Pound JL. An advance triage system. Accid Emerg Nurs 2002; 10: 10-6. PM 11998578 (level 4).

172. Maeda S. Test in the overtime outpatient department. Kyukyuigaku. 2010;34:982-4 (in Japanese) (Level 5).

173. Evennett NJ, Petrov MS, Mittal A, et al. Systematic review and pooled estimates for the diagnostic accuracy of serological markers for intestinal ischemia. World $\mathrm{J}$ Surg. 2009;33:1374-83. 